\title{
We will be known by the tracks we leave behind: Exotic lithic raw materials, mobility and social networking among the Côa Valley foragers (Portugal)
}

\author{
Thierry Aubry ${ }^{\mathrm{a}, *}$, Luís Luís ${ }^{\mathrm{a}}$, Javier Mangado Llach ${ }^{\mathrm{b}}$, Henrique Matias ${ }^{\mathrm{c}}$ \\ ${ }^{a}$ Côa Parque, Fundação para a Salvaguarda e Valorização do Vale do Côa, Rua do Museu, 5150-610 Vila Nova de Foz Côa, Portugal \\ ${ }^{\mathrm{b}}$ Departament de Prehistòria, Història Antiga i Arqueologia, 1er pis, Despatx 1011SERP (Seminari d'Estudis i Recerques Prehistòriques), \\ Fac. de Geografia i Història, Universitat de Barcelona, C/Montalegre 6-8, 08001 BCN, Spain \\ ${ }^{\mathrm{C}}$ UNIARQ Centro de Arqueologia, Universidade de Lisboa, Faculdade de Letras, Alameda da Universidade, 1600-214 Lisboa, Portugal
}

\section{A R T I C L E I N F O}

\section{Article history:}

Received 23 January 2012

Revision received 19 May 2012

Available online $\mathrm{xxxx}$

\section{Keywords:}

Toolstone sources

Upper Palaeolithic

Iberia

Land-use

Mobility

Open-air rock art

\begin{abstract}
A B S T R A C T
The aim of this study is to establish different socio-cultural models based on lithic raw material sourcing integrated with regional rock art distributions to infer social behaviours within the context of central Iberian Upper Palaeolithic societies. Specifically, we focus on Upper Palaeolithic hunter-gatherer mobility and social interaction in the Côa Valley. This study relies on a knowledge of regional geology and field work to identify the sources of the lithic raw material found at Côa Valley Upper Palaeolithic sites. We expose the context of use and discard of flint, which is naturally absent from the region, and other local materials. From this, we present a GIS based analysis concerning space, time and least-cost paths travelled. This analysis, along with the environmental data available, forms a baseline to evaluate different anthropological models on hunter-gatherer lithic use, mobility, and social networks. We conclude with a three-level model for social interaction, in the context of aggregation activities between different social meta-groups, which involves embedded procurement, long-distance raw material exchange, and symbolic community, reflected in the largest known open-air Upper Palaeolithic rock art concentration.
\end{abstract}

(c) 2012 Elsevier Inc. All rights reserved.
We will be known by the tracks we leave behind.

Dakota proverb

\section{Introduction}

The interpretation of the spatial of lithic raw material sources used to reconstruct hunter-gatherer landscapes is still a matter of debate among many researchers and multiple interpretations of the same data are often advanced. Moreover, when stressing the importance of raw materials, one should never forget that they reflect a small, even if well preserved, part of the foraging activities of past hunter-gatherer groups. Other resources, namely biotic, that may have played an even bigger role than lithic raw materials, have since been subjected to taphonomic filters, and are inaccessible in our analysis.

In an ecological perspective, lithic raw material sourcing can be used to determine past demography, since, the exploited space should reflect environmental resource supply and forager needs (Winterhalder, 2001; Hamilton et al., 2007). Besides being informative of prehistoric population size and territories, raw material stud-

\footnotetext{
* Corresponding author. Fax: +351 279769270.

E-mail addresses: thaubry@sapo.pt (T. Aubry), lluis.pavc@igespar.pt (L. Luís), mangado@ub.edu (J. Mangado Llach), hamatias@gmail.com (H. Matias).
}

ies can also inform us of changes in pattern of resource exploitation through time - a reflection of forager economic and seasonal variability (Demars, 1982; Féblot-Augustins, 1997). With this approach the distances of displacement, raw material quality and quantities present at a site, and the documentation of different lithic reduction phases leading to artefact abandonment (Geneste, 1985) are essencial to reconstruct forager's mobility. This approach has not only been applied to Anatomically Modern Humans but also to Neanderthal populations (Féblot-Augustins, 1999; Fernandes et al., 2008).

However, when dealing with an eminently social species, territory use, notably reflected in long-distance lithic raw material sources may express non-utilitarian purposes. The raw materials in an archaeological assemblage can therefore be the result, not only of direct procurement (Binford, 1980), but also exchange (Gamble, 1999). This is the case of long-distance raw materials, which have been used to define social networks fundamental for human survival (Whallon, 2006). In this case, lithic raw material could be the expression of immaterial behaviours, such as information exchange.

The aim of this study is to establish different sociocultural models from lithic raw material sourcing and to infer social behaviours within the context of central Iberian Upper Palaeolithic societies, integrating it with rock art distribution. We analyse the role of cultural vs. lithic resource constraints in the choice of reduction sequences, the mobility patterns of these peoples and their cultural 
geography. For this purpose we have conducted field surveys to define a raw material data set, macroscopic and microscopic observations were performed to better characterise the geological sources, and a Geographic Information Systems (GISs) approach was used to analyse distances and paths to the lithic sources used. Finally, we compared the Côa Valley toolstone supply with settlement data from sedimentary areas where flint is naturally present, some of which also are used in the Côa Valley, and interpreted the observed archaeological reality in the light of ethnographic data.

\section{Material and methods}

\section{Côa Valley Upper Palaeolithic setting and occupation sequence}

Geomorphologically the core of the Iberian Peninsula corresponds to a large plateau, the Meseta, a Cenozoic peneplanation structural surface (Ferreira, 1978), which is divided in two by the Central Mountain System (Fig. 1a). To the west, the Northern pla- teau is limited by the SSW/NNE Manteigas-Vilariça-Bragança fault system, separating it from the Portuguese Western Mountains (Ferreira, 1978). Conditioned by this tectonic structure (Aubry et al., 2012), the Côa river runs deeply gorged a few km east of this limit (Fig. 1b).

It was in this area, notably in the last $17 \mathrm{~km}$ of the Côa Valley, that, during the 1990s, stylistically Upper Palaeolithic open-air rock art was discovered (Fig. 1b), in a region where no evidence of Upper Palaeolithic human occupation had ever been reported before (Zilhão et al., 1995). Subsequently, the discovery and study of an archaeological context for the rock art has enabled us to establish a significant occupation in this region of Iberia, during several periods of the Upper Palaeolithic (Zilhão, 1997; Aubry, 2001, 2002, 2009), partially contradicting the accepted logistic hunting sites model for the Iberian hinterland's scarce occurrences (Davidson, 1986). More than 15 archaeological sites have since been identified in the Côa Valley (10 of which were tested or excavated) for these periods over less than $400 \mathrm{~km}^{2}$ along the river's

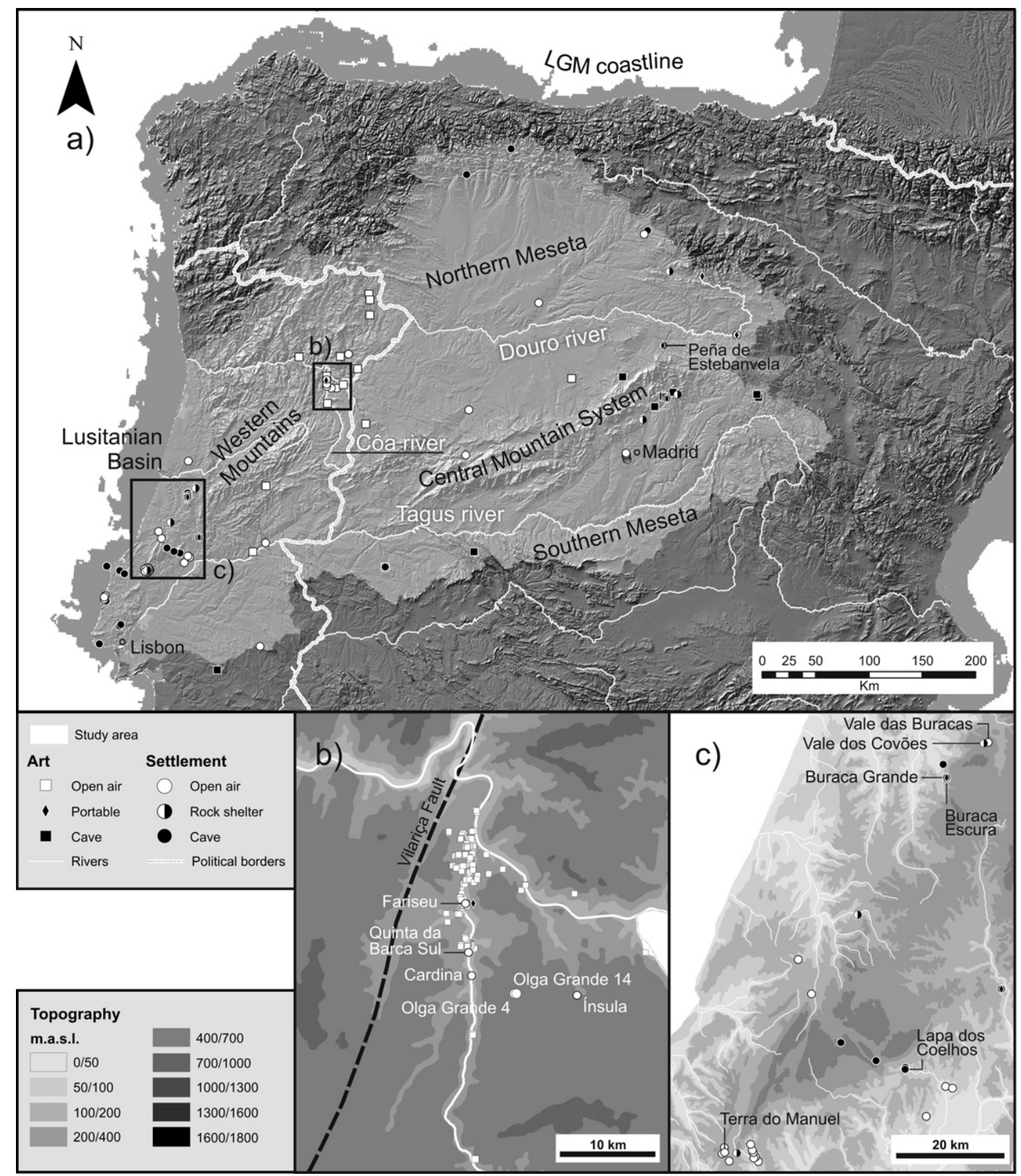

Fig. 1. Upper Palaeolithic settlement, rock art and mobile art sites in the study area (a) with detailed vision for the Côa Valley (b) and Portuguese Estremadura (c). 
nine last kilometres on both margins of the valley bottom, and on the granitic bedrock of the surrounding plateau (Aubry et al., 2002; Aubry and Sampaio, 2008a; Aubry et al., 2010).

In Portugal, Upper Palaeolithic remains present some peculiarities when compared to central and northern European contexts, in terms of animal species exploited, bone and lithic tool categories, as well as operative schemes (Zilhão, 1997; Bicho, 2000; Almeida, 2000; Aubry, 2009). Contrarily to most of European Upper Palaeolithic lithic stone-tool assemblages all the Portuguese series show systematic and profuse use of quartzite (Pereira, 2010) and quartz. Nevertheless, the typology of diagnostically Solutrean lithic points and of the retouched bladelets recovered on the Côa Valley sites has permitted, by comparison with other dated Portuguese lithic series, the establishment of a chronostratigraphic framework of the Upper Palaeolithic sequence of occupation, confirmed by radiometric ages obtained at four sites, by luminescence on burnt quartz and quartzite pebbles and sediments (Valladas et al., 2001; Mercier et al., 2001, 2006) and by ${ }^{14} \mathrm{C}$ (Aubry, 2009) (Table 1 ).

These sites reveal the discard of small quantities of raw materials formed in sedimentary environments absent from the regional geological context (Mangado Llach, 2002, 2004; Aubry and Mangado Llach, 2003a, 2003b, 2006; Aubry et al., 2002, 2003, 2004). This environmental constraint to the Upper Palaeolithic settlement in the area provides us a unique opportunity to study the origin and displacement of these materials, which attest long-distance connections with other areas of the Iberian Peninsula, namely Central Spain and Central Portugal, that hint at mobility patterns and long-distance social relations.

A relative chronology of the different phases of the Côa Valley Upper Palaeolithic occupation sequence was established from stratigraphic sequences identified at five sites, where a geoarchaeolological approach was applied (Aubry et al., 2010). Radiometric ages were obtained at four Côa Valley sites, by luminescence applied on burnt quartz and quartzite pebbles and by Optically Stimulated Luminescence on sediments, at Cardina 1, Olga Grande 4, Quinta da Barca Sul and Fariseu (Valladas et al., 2001; Mercier et al., 2001, 2006) (Table 1). The acid soils developed on schist and granite bedrock of the region do not favour the preservation of macro organic remains. However, Upper Palaeolithic radiocarbon ages were obtained on bones and teeth recovered in stratigraphic level 4 of Fariseu (Aubry et al., 2007; Aubry and Sampaio, 2008b; Aubry, 2009), where faunal remains are unusually preserved (Gabriel and Béarez, 2009). Stratigraphic level 9 of this site, bearing a charcoal fragment dated to $19,020 \pm 80{ }^{14} \mathrm{C}$ years $\mathrm{BP}$ (GrA-40167; 22,581-23,174 cal years BP) by AMS demonstrates the potential conservation of Last Glacial Maximum (LGM) macro organic materials preserved in alluvial deposits. The ages obtained at the four sites have confirmed the typological attribution of the stone tool assemblages found therein and indicate human presence during several phases in the 31,000-12,000 BP interval (Table 1).

\section{Raw material characterisation and technological analysis}

The study of lithic raw material procurement was based on systematic descriptions and comparisons of archaeological and geological samples. The latter were collected specifically for this study during field work based on geological data provided by the Instituto Geológico e Mineiro de Portugal (Carta Geológica de Portugal, Scale 1:50,000) and Instituto Geológico y Minero de España (Mapa Geológico de España, Scale 1:50,000) (see Table 2). The geological data set was established during surveys undertaken by the authors, with the collaboration of Jorge Davide Sampaio and André Santos, between 1997 and 2011.

Archaeological and geological samples were described at different scales of analysis (macroscopic with stereomicroscope and microscopic thin sections) (Mangado Llach, 2002, 2004; Aubry and Mangado Llach, 2006). A total of 93,010 lithic artefact remains recovered at 11 sites were studied and data by $C$. Gameiro from the Lapa dos Coelhos cave (3932 lithic artefact remains) were also examined (Tables 1, 3 and 4). The geological samples from the Duero/Douro and Tagus basins were studied in terms of texture and structure, mineralogical and palaeontological content, defining 59 categories (Mangado Llach, 2002, 2004; Aubry and Mangado Llach, 2003a, 2003b, 2006, Table 2), following the genetic and gytological description proposed by Fernandes et al. (2008). The preliminary systematic macroscopic examination was complemented by a petrographic analysis (mineralogy, texture and fossil content) of archaeological and geological samples collected from the different sedimentary basins (Mangado Llach, 2002, 2004).

Lithic assemblages were classified into lithological types and technological classes corresponding to reduction sequences starting with blocks, fragments from veins of siliceous rock, nodules or pebbles, in order to establish the production processes represented for each different raw material, their techniques and purposes and blank selection for retouched tools (Leroi-Gourhan and Brézillon, 1966). An economical interpretation of the data is proposed, based on the production sequences present and absent for each lithological category, as well as on the reconstruction of the techno-morphological objectives underlying the deliberate production of some flake blanks not designed for retouch (Aubry, 2009). The litho-technological information was complemented by use-wear analysis of a selected sample, representative of the retouched tools and blanks (Aubry and Araújo Igreja, 2009).

\section{Geographical approach}

Since resources are sparse and unequally distributed over the landscape, mobility is an essential feature of ancient and presentday hunter-gatherer societies. Used in association with archaeological data, GIS tools, notably cost and time surface analysis, are probably one of the most objective ways to determine routes and tracks that may have structured past hunter-gatherer landscapes.

These tools can determine the effort to travel across a certain landscape and optimal paths between two locations, taking into account physical obstacles to movement, namely topography and rivers (Wheatley and Gillings, 2002; van Leusen, 2002). Nevertheless, they present several limitations, connected with the different algorithms used (isotropic or anisotropic, energy or time models, etc.), that may not accurately translate the reality of human movement, but mainly connected with the fact that they are not suited to account for cultural or social constraints to movement, such as liminal areas. These analyses are also strongly dependent on the accuracy and resolution of the Digital Elevation Model (DEM) in which they are based (Wheatley and Gillings, 2002). Therefore, cost surface analysis should be taken not as an absolute but instead as a probabilistic approach to the time and space travelled between two locations (van Leusen, 2002).

Despite its limitations, cost surface analysis represents a major breakthrough, freeing spatial analysis from Cartesian space. It recognises the fact that a straight line is not always the shortest distance between two points. After determining that certain objects travelled from known sources to the Côa Valley, our goal was to probabilistically assess the routes they may have travelled, and the time it would take to effect their transportation. This approach could get us closer to the perception of the landscape held by the human groups involved in this transport.

In our analysis we've used the Shuttle Radar Topographic Mission (SRTM) 30 arc-second elevation data (Farr et al., 2007) in order to build our DEM. To reconstruct Last Glacial Maximum coastlines, which were $120 \mathrm{~m}$ lower than present day sea level (Lambeck and Chappell, 2001), we've also used bathymetry data from ETOPO1 
Table 1

Upper Palaeolithic archaeological lithic series of the Côa Valley and Portuguese Estremadura considered in this study.

\begin{tabular}{|c|c|c|c|c|c|c|c|c|c|c|c|c|}
\hline Site & Environment & Latitude $(\mathrm{N})$ & $\begin{array}{l}\text { Longitude } \\
\text { (W) }\end{array}$ & m.a.s.l. & Layer & Age (yrs BP) & $\begin{array}{l}{ }^{14} \mathrm{C} \text { Calibrated } \\
\text { Age }(\text { cal yr BP) }\end{array}$ & Lab code & $\begin{array}{l}\text { Dating } \\
\text { method }\end{array}$ & $\begin{array}{l}\text { Cultural } \\
\text { attribution }\end{array}$ & References & \\
\hline \multirow[t]{5}{*}{ Olga Grande 4} & 4 Open air & $40^{\circ} 58^{\prime} 10.44^{\prime \prime}$ & $7^{\circ} 03^{\prime} 23.43^{\prime \prime}$ & 510 & 3 & $26,800 \pm 2300$ & - & $\begin{array}{l}\text { GYF-OG } 4 \\
\text { no. } 4\end{array}$ & $\mathrm{TL}$ & Gravettian & $\begin{array}{l}\text { Valladas } \\
\text { et al. (2001) }\end{array}$ & Côa Valley \\
\hline & & & & & 3 & $27,200 \pm 2300$ & - & $\begin{array}{l}\text { GYF-OG4 } \\
\text { no. } 1\end{array}$ & $\mathrm{TL}$ & Gravettian & $\begin{array}{l}\text { Valladas } \\
\text { et al (2001) }\end{array}$ & \\
\hline & & & & & 3 & $28,500 \pm 2300$ & - & $\begin{array}{l}\text { GYF-OG4 } \\
\text { no. } 3\end{array}$ & $\mathrm{TL}$ & Gravettian & $\begin{array}{l}\text { Valladas } \\
\text { et al. (2001) }\end{array}$ & \\
\hline & & & & & 3 & $30,000 \pm 2400$ & - & $\begin{array}{l}\text { GYF-OG4 } \\
\text { no. } 2\end{array}$ & $\mathrm{TL}$ & Gravettian & $\begin{array}{l}\text { Valladas } \\
\text { et al. (2001) }\end{array}$ & \\
\hline & & & & & 3 & $31,000 \pm 2500$ & - & $\begin{array}{l}\text { GYF-OG4 } \\
\text { no. } 5\end{array}$ & $\mathrm{TL}$ & Gravettian & $\begin{array}{l}\text { Valladas } \\
\text { et al. (2001) }\end{array}$ & \\
\hline \multirow[t]{2}{*}{$\begin{array}{l}\text { Olga Grande } \\
14\end{array}$} & Open air & $40^{\circ} 58^{\prime} 10.19^{\prime \prime}$ & $7^{\circ} 03^{\prime} 17.99^{\prime \prime}$ & 510 & $2 c$ & - & - & - & Typology & $\begin{array}{l}\text { Proto- } \\
\text { Solutrean }\end{array}$ & $\begin{array}{l}\text { Aubry } \\
\text { (2009) }\end{array}$ & \\
\hline & & & & & 3 & - & - & - & Typology & Gravettian & $\begin{array}{l}\text { Aubry } \\
\text { (2009) }\end{array}$ & \\
\hline \multirow[t]{12}{*}{ Cardina 1} & Open air & $40^{\circ} 58^{\prime} 46.11^{\prime \prime}$ & $7^{\circ} 03^{\prime} 23.43^{\prime \prime}$ & 165 & 4.6 & - & - & - & Typology & $\begin{array}{l}\text { Upper } \\
\text { Magdalenian }\end{array}$ & $\begin{array}{l}\text { Aubry } \\
\text { (2009) }\end{array}$ & \\
\hline & & & & & 4.7 & - & - & - & Typology & $\begin{array}{l}\text { Upper } \\
\text { Magdalenian }\end{array}$ & $\begin{array}{l}\text { Aubry } \\
\text { (2009) }\end{array}$ & \\
\hline & & & & & 4.8 & - & - & - & Typology & $\begin{array}{l}\text { Magdalenian/ } \\
\text { Solutrean }\end{array}$ & Aubry, 2009 & \\
\hline & & & & & 4.9 & - & - & - & Typology & $\begin{array}{l}\text { Magdalenian/ } \\
\text { Solutrean }\end{array}$ & $\begin{array}{l}\text { Aubry } \\
(2009)\end{array}$ & \\
\hline & & & & & 4.10 & $20,700 \pm 1300$ & - & $\begin{array}{l}\text { GYF-Card } \\
\text { no. } 5\end{array}$ & $\mathrm{TL}$ & Solutrean & $\begin{array}{l}\text { Valladas } \\
\text { et al. (2001) }\end{array}$ & \\
\hline & & & & & 4.10 & $23,400 \pm 1500$ & - & $\begin{array}{l}\text { GYF-Card } \\
\text { no. } 8\end{array}$ & $\mathrm{TL}$ & $\begin{array}{l}\text { Proto- } \\
\text { Solutrean }\end{array}$ & $\begin{array}{l}\text { Valladas } \\
\text { et al. (2001) }\end{array}$ & \\
\hline & & & & & 4.10 & $26,500 \pm 1800$ & - & $\begin{array}{l}\text { GYF-Card } \\
\text { no. } 10\end{array}$ & $\mathrm{TL}$ & $\begin{array}{l}\text { Recent } \\
\text { Gravettian }\end{array}$ & $\begin{array}{l}\text { Valladas } \\
\text { et al. (2001) }\end{array}$ & \\
\hline & & & & & 4.10 & $27,000 \pm 1800$ & - & $\begin{array}{l}\text { GYF-Card } \\
\text { no. } 9\end{array}$ & $\mathrm{TL}$ & $\begin{array}{l}\text { Recent } \\
\text { Gravettian }\end{array}$ & $\begin{array}{l}\text { Valladas } \\
\text { et al. (2001) }\end{array}$ & \\
\hline & & & & & 4.10 & $27,800 \pm 1500$ & - & $\begin{array}{l}\text { GYF-Card } \\
\text { no. } 3\end{array}$ & $\mathrm{TL}$ & $\begin{array}{l}\text { Recent } \\
\text { Gravettian }\end{array}$ & $\begin{array}{l}\text { Valladas } \\
\text { et al. (2001 }\end{array}$ & \\
\hline & & & & & 4.10 & $28,000 \pm 2100$ & - & $\begin{array}{l}\text { GYF-Card } \\
\text { no. } 4\end{array}$ & $\mathrm{TL}$ & $\begin{array}{l}\text { Recent } \\
\text { Gravettian }\end{array}$ & $\begin{array}{l}\text { Valladas } \\
\text { et al. (2001) }\end{array}$ & \\
\hline & & & & & 4.10 & $30,100 \pm 1500$ & - & $\begin{array}{l}\text { GYF-Card } \\
\text { no. } 7\end{array}$ & $\mathrm{TL}$ & $\begin{array}{l}\text { Recent } \\
\text { Gravettian }\end{array}$ & $\begin{array}{l}\text { Valladas } \\
\text { et al. ( } 2001\end{array}$ & \\
\hline & & & & & 4.11 & - & - & - & Typology & $\begin{array}{l}\text { Recent } \\
\text { Gravettian }\end{array}$ & Aubry, 2009 & \\
\hline Ínsula & Open air & $40^{\circ} 58^{\prime} 10.23^{\prime \prime}$ & 659'28.99" & 310 & 2 & - & - & - & Typology & $\begin{array}{l}\text { Recent } \\
\text { Gravettian }\end{array}$ & $\begin{array}{l}\text { Aubry } \\
\text { (2009) }\end{array}$ & \\
\hline \multirow[t]{3}{*}{$\begin{array}{l}\text { Quinta da } \\
\text { Barca Sul }\end{array}$} & Open air & $40^{\circ} 59^{\prime} 54.01^{\prime \prime}$ & $7^{\circ} 06^{\prime} 14.09^{\prime \prime}$ & 143 & 3 & $11,600 \pm 1200$ & - & $\begin{array}{l}\text { GYF-QBS } \\
\text { no. } 3\end{array}$ & $\mathrm{TL}$ & $\begin{array}{l}\text { Final } \\
\text { Magdalenian }\end{array}$ & $\begin{array}{l}\text { Valladas } \\
\text { et al. (2001) }\end{array}$ & \\
\hline & & & & & 3 & $11,900 \pm 1100$ & - & $\begin{array}{l}\text { GYF-QBS } \\
\text { no. } 4\end{array}$ & $\mathrm{TL}$ & $\begin{array}{l}\text { Final } \\
\text { Magdalenian }\end{array}$ & $\begin{array}{l}\text { Valladas } \\
\text { et al. (2001) }\end{array}$ & \\
\hline & & & & & 3 & $12,700 \pm 1000$ & - & $\begin{array}{l}\text { GYF-QBS } \\
\text { no. } 2\end{array}$ & $\mathrm{TL}$ & $\begin{array}{l}\text { Final } \\
\text { Magdalenian }\end{array}$ & $\begin{array}{l}\text { Valladas } \\
\text { et al. (2001) }\end{array}$ & \\
\hline \multirow[t]{7}{*}{ Fariseu } & Open air & $41^{\circ} 02^{\prime} 16.64^{\prime \prime}$ & $7^{\circ} 06^{\prime} 36.53^{\prime \prime}$ & 125 & 4 & $9830 \pm 130$ & $11,096-11,539$ & $\begin{array}{l}\text { Ua- } \\
32645\end{array}$ & ${ }^{14} \mathrm{C}$ AMS & $\begin{array}{l}\text { Final } \\
\text { Magdalenian }\end{array}$ & $\begin{array}{l}\text { Aubry } \\
\text { (2009) }\end{array}$ & \\
\hline & & & & & 4 & $11,800 \pm 900$ & - & $\begin{array}{l}\text { GYF-FAR } \\
\text { no. } 8\end{array}$ & TL/OSL & $\begin{array}{l}\text { Final } \\
\text { Magdalenian }\end{array}$ & $\begin{array}{l}\text { Mercier } \\
\text { et al. (2006) }\end{array}$ & \\
\hline & & & & & 4 & $11,000 \pm 1100$ & - & $\begin{array}{l}\text { GYF-FAR } \\
\text { no. } 17\end{array}$ & TL/OSL & $\begin{array}{l}\text { Final } \\
\text { Magdalenian }\end{array}$ & $\begin{array}{l}\text { Mercier } \\
\text { et al. (2006) }\end{array}$ & \\
\hline & & & & & 4 & $10,800 \pm 1700$ & - & $\begin{array}{l}\text { GYF-FAR } \\
\text { no. } 2\end{array}$ & $\mathrm{TL}$ & $\begin{array}{l}\text { Final } \\
\text { Magdalenian }\end{array}$ & $\begin{array}{l}\text { Mercier } \\
\text { et al. (2006) }\end{array}$ & \\
\hline & & & & & 4 & $10,510 \pm 40$ & $12,287-12,621$ & $\begin{array}{l}\text { Beta- } \\
213130\end{array}$ & ${ }^{14} \mathrm{C}$ AMS & $\begin{array}{l}\text { Final } \\
\text { Magdalenian }\end{array}$ & $\begin{array}{l}\text { Aubry } \\
(2009)\end{array}$ & \\
\hline & & & & & 9 & $19,020 \pm 80$ & $22,581-23,174$ & $\begin{array}{l}\text { GrA- } \\
40167\end{array}$ & ${ }^{14} \mathrm{C}$ AMS & Solutrean & $\begin{array}{l}\text { Aubry } \\
\text { (2009) }\end{array}$ & \\
\hline & & & & & 10 & - & - & - & Typology & $\begin{array}{l}\text { Gravettian/ } \\
\text { Solutrean }\end{array}$ & $\begin{array}{l}\text { Aubry } \\
\text { (2009) }\end{array}$ & \\
\hline \multirow[t]{2}{*}{$\begin{array}{l}\text { Buraca } \\
\text { Grande }\end{array}$} & Cave & $39^{\circ} 58^{\prime} 51.59^{\prime \prime}$ & $8^{\circ} 33^{\prime} 0.69^{\prime \prime}$ & 256 & $\begin{array}{l}8 c / \\
9 a\end{array}$ & - & - & - & Typology & Solutrean & $\begin{array}{l}\text { Aubry et al. } \\
\text { (1997) }\end{array}$ & \multirow[t]{4}{*}{$\begin{array}{l}\text { Portuguese } \\
\text { Estremadura }\end{array}$} \\
\hline & & & & & $9 b$ & $23,920 \pm 300$ & $28,355-29,255$ & $\begin{array}{l}\text { GifA- } \\
93048\end{array}$ & ${ }^{14} \mathrm{C}$ AMS & Gravettian & $\begin{array}{l}\text { Aubry et al. } \\
\text { (1997) }\end{array}$ & \\
\hline Buraca Escura & Cave & $39^{\circ} 58^{\prime} 47.55^{\prime \prime}$ & $8^{\circ} 33^{\prime} 7.65^{\prime \prime}$ & 223 & $2 \mathrm{ab}$ & $21,820 \pm 200$ & $25,582-26,634$ & $\begin{array}{l}\text { OxA- } \\
5524\end{array}$ & ${ }^{14} \mathrm{C}$ AMS & $\begin{array}{l}\text { Recent } \\
\text { Gravettian }\end{array}$ & $\begin{array}{l}\text { Aubry et al. } \\
(2001)\end{array}$ & \\
\hline $\begin{array}{l}\text { Vale das } \\
\text { Buracas }\end{array}$ & Open air & $40^{\circ} 2^{\prime} 46.01^{\prime \prime}$ & $8^{\circ} 29^{\prime} 45.10^{\prime \prime}$ & 290 & $\begin{array}{l}3 \mathrm{~b}, \\
3 / 4\end{array}$ & - & - & - & Typology & Gravettian & $\begin{array}{l}\text { Aubry et al. } \\
(2008)\end{array}$ & \\
\hline
\end{tabular}


Table 1 (continued)

\begin{tabular}{|c|c|c|c|c|c|c|c|c|c|c|c|}
\hline Site & Environment & Latitude $(\mathrm{N})$ & $\begin{array}{l}\text { Longitude } \\
\text { (W) }\end{array}$ & m.a.s.l. & Layer & Age (yrs BP) & $\begin{array}{l}{ }^{14} \mathrm{C} \text { Calibrated } \\
\text { Age (cal yr BP) }\end{array}$ & Lab code & $\begin{array}{l}\text { Dating } \\
\text { method }\end{array}$ & $\begin{array}{l}\text { Cultural } \\
\text { attribution }\end{array}$ & References \\
\hline \multirow[t]{4}{*}{$\begin{array}{l}\text { Vale dos } \\
\text { Covões }\end{array}$} & Rock shelter & $40^{\circ} 2^{\prime} 51.74^{\prime \prime}$ & $8^{\circ} 29^{\prime} 57.83^{\prime \prime}$ & 275 & 3 & - & - & - & Typology & $\begin{array}{l}\text { Upper } \\
\text { Magdalenian }\end{array}$ & $\begin{array}{l}\text { Aubry et al. } \\
\text { (2008) }\end{array}$ \\
\hline & & & & & 4 & - & - & - & Typology & $\begin{array}{l}\text { Upper } \\
\text { Magdalenian }\end{array}$ & $\begin{array}{l}\text { Aubry et al. } \\
(2008)\end{array}$ \\
\hline & & & & & 5 & $10,540 \pm 95$ & $12,251-12,643$ & $\begin{array}{l}\text { Ua- } \\
33479\end{array}$ & ${ }^{14} \mathrm{C}$ AMS & $\begin{array}{l}\text { Upper } \\
\text { Magdalenian }\end{array}$ & $\begin{array}{l}\text { Klaric et al. } \\
(2009)\end{array}$ \\
\hline & & & & & 5 & $12,340 \pm 50$ & $14,174-14,853$ & $\begin{array}{l}\text { Beta- } \\
201013\end{array}$ & ${ }^{14} \mathrm{C}$ AMS & $\begin{array}{l}\text { Upper } \\
\text { Magdalenian }\end{array}$ & $\begin{array}{l}\text { Klaric et al. } \\
(2009)\end{array}$ \\
\hline $\begin{array}{l}\text { Lapa dos } \\
\quad \text { Coelhos }\end{array}$ & Cave & $39^{\circ} 30^{\prime} 17.41^{\prime \prime}$ & $8^{\circ} 36^{\prime} 55.19^{\prime \prime}$ & 85 & 3 & $11,660 \pm 60$ & $13,409-13,683$ & $\begin{array}{l}\text { GrN- } \\
18376\end{array}$ & ${ }^{14} \mathrm{C}$ AMS & $\begin{array}{l}\text { Upper } \\
\text { Magdalenian }\end{array}$ & $\begin{array}{l}\text { Almeida } \\
\text { et al. (2004) }\end{array}$ \\
\hline \multirow[t]{3}{*}{$\begin{array}{l}\text { Terra do } \\
\quad \text { Manuel }\end{array}$} & Open air & $39^{\circ} 20^{\prime} 41.72^{\prime \prime}$ & $8^{\circ} 59^{\prime} 30.77^{\prime \prime}$ & 110 & $2 \mathrm{~s}$ & $21,770 \pm 210$ & $25,456-26,594$ & $\begin{array}{l}\text { ETH- } \\
6038\end{array}$ & ${ }^{14} \mathrm{C}$ AMS & $\begin{array}{l}\text { Proto- } \\
\text { Solutrean }\end{array}$ & $\begin{array}{l}\text { Zilhão } \\
\text { (1997) }\end{array}$ \\
\hline & & & & & $2 \mathrm{~s} 1$ & - & - & - & Typology & $\begin{array}{l}\text { Proto- } \\
\text { Solutrean }\end{array}$ & $\begin{array}{l}\text { Almeida } \\
\text { et al. (2010) }\end{array}$ \\
\hline & & & & & $2 \mathrm{~s} 2$ & - & - & - & Typology & $\begin{array}{l}\text { Recent } \\
\text { Gravettian }\end{array}$ & $\begin{array}{l}\text { Almeida } \\
\text { et al. (2010) }\end{array}$ \\
\hline
\end{tabular}

a Calibration used CalPal with the Calcurve CalPal_2007_HULU (www.calpal-online.de). All the errors are 1-sigma.

(Amante and Eakins, 2009). Elevation data was projected into a metric coordinate system (ETRS 1989 UTM Zone 30N).

From this DEM we've estimated anisotropic distances to each settlement site analysed, using ArcGIS's PathDistance function, where the vertical factor was determined using Tobler's (1993) hiking function, as proposed by Tripcevich (2007, 2009). This function converts space into time. Therefore, our distances are always determined in minimum time spent, not kilometres walked, thus closer to cognitive space. In our analysis we have not accounted for vegetation or watercourses as an obstacle to movement. Tobler's hiking function is based on empirical data provided by Imhof (1950). Therefore, it does not account for vegetation obstacles to displacement, which, nevertheless we consider negligible during the LGM, when herbaceous scrublands dominated (Zilhão, 1997; Haws, 2011). Although crossing rivers can be a limitation, they must nevertheless be crossed, and no model at the scale we've worked could account for natural fording places. A problem could arise if the least-cost paths would follow mainly stream lines, since they tend to be gently sloped lines (Fábrega Álvarez and Parcero Oubiña, 2007), but, once the least costs were produced, careful examination showed that was not the case. From these anisotropic cost surfaces, least-cost paths were generated from each site to each source presented in each archaeological layer. In order to calculate used resource areas, polygons were produced with ET Geowizards function, having the raw material sources location used as vertices.

\section{Results}

\section{Geological setting and differential toolstone sources potential}

The Duero/Douro and Tagus River Basins cover one third of the total Iberian Peninsula area, integrating a Mesozoic marine basin (Lusitanian), a Cenozoic basin in the continental crust (Duero, Madrid and Lower Tagus), and structural uplifts in the continental crust (Central Mountain System and Western Mountains) (Ribeiro et al., 1979; Kullberg et al., 2006; de Vicente et al., 2011).

Direct sampling and macroscopic analysis of siliceous rocks collected at 79 locations, and the microscopic petrographic and palaeontological description of siliceous rocks from 27 more outcrops of the Duero/Douro and Tagus basins allowed us to establish a precise and representative data set of the potential lithic raw material sources available in primary and secondary deposits (Table 2 and Fig. 2). A long-term survey has shown there are geographical dense clusters of outcrops where siliceous rocks nodules are abundant, available in large sizes and homogeneously spread through the landscape, but there are other regions where usable sources are restricted spatially or scarce among siliceous rocks not as suitable for knapping. Experimental knapping on the different siliceous rocks collected allowed us to establish the quality range of each source in terms of its adaptation to produce bladelets (evaluated from 1 [low quality] to 10 [high quality] in Table 2). The geographical distribution of each petrologic variety enabled us to identify raw material sources, and the systematic macroscopic and microscopic analyses in thin section of selected archaeological pieces permitted us to establish the ones used in the Côa Valley Upper Palaeolithic sites (Mangado Llach, 2002; Aubry, 2009).

Coarse-grain Armorican metaquartzites (Lower Ordovician) are ubiquitous rocks in the Central-Iberian Zone of the Iberian Massif (McDougall et al., 1987) found as narrow crests. These erosion resistant rocks are one of the most common materials in the composition of the coarse-grained Duero/Douro and Tagus fluvial deposits. Conglomerates, sandstones, and sandy mudstone varieties are less frequent but exist as consolidated fluvial deposits, dating from the Final Cretaceous to the Palaeocene in the Duero/Douro basin (Mediavilla and Dabrio, 1986; Blanco et al., 2008). Quartz is present in its different mineralogical forms, as hydrothermal vein fillings, in the joint/fractures resulting from Variscan tectonic events, and in secondary position in detrital deposits.

Within this area, flint occurs in three main depositional environments. The first consists of syn-diagenetic siliceous precipitations formed in open or barrier reef continental shelf environments identified along the stratigraphic Mesozoic sequence of the Lusitanian basin. The evolution of the western Iberian margin, closely associated with the first opening phases of the North Atlantic, ranges from the Late Triassic to the Early Cretaceous, and its evolution was conditioned by inherited structures from the Variscan basement (Kullberg et al., 2006). The second, more circumscribed, flint source type appears in Miocene lacustrine environment of the Central Mountain System's northern and southern margins (Bustillo, 1976; Bustillo and Pérez-Jiménez, 2005; Armenteros, 2000). Finally, silcrete composed by amorphous silica in origin, evolving to opal-CT, occurs in dispersed and restrict outcrops of the northern and southern Meseta (Molina Ballesteros et al., 1997; Blanco et al., 2008), and in the Lusitanian basin (Proença Cunha, 2000).

Most of these sources are geographically located and geologically well defined by their sedimentary facies, detrital and palaeontological content (Table 2 and Fig. 2). However, several source locations could provide the same raw material type. In this case, we've used the most parsimonious hypothesis and assumed the 
Fine-grained siliceous rocks sources sampled and described in the Douro and Tagus basins.

\begin{tabular}{|c|c|c|c|c|c|c|c|c|c|c|}
\hline \multirow[t]{2}{*}{ Code } & \multirow{2}{*}{$\begin{array}{l}\text { Genetic } \\
\text { Type }^{\mathrm{a}}\end{array}$} & \multirow{2}{*}{$\begin{array}{l}\text { Gytologic } \\
\text { Types }^{\mathrm{b}}\end{array}$} & \multirow{2}{*}{$\begin{array}{l}\text { Sedimentary } \\
\text { Structure }^{\mathrm{c}}\end{array}$} & \multirow[t]{2}{*}{ Texture $^{\mathrm{d}}$} & \multirow[t]{2}{*}{ Mineralogy $^{\mathrm{e}}$} & \multicolumn{2}{|l|}{ Constituents } & \multirow[t]{2}{*}{ Environment $^{\mathrm{h}}$} & \multirow[t]{2}{*}{ Quality $^{\mathrm{i}}$} & \multirow[t]{2}{*}{ Refs. $^{j}$} \\
\hline & & & & & & Skeletal grain/bioclasts ${ }^{\mathrm{f}}$ & Porosity and non-skeletal ${ }^{g}$ & & & \\
\hline L1 & Ca-1 & 1 & LAM & MUD & $\mathrm{mQ}, \mathrm{MQ}$ & NOTS & NOTS & MAR & 4 & 1,2 \\
\hline A1 & TJ-1 & 1 & - & MUD & CQ-mQ (70\%), MQ (10\%), CAL-LF (10\%) & - & MOL, OF, FEL, MOS, Q-TER & MAR-CS & 4 & $\begin{array}{l}1,2 \\
3\end{array}$ \\
\hline A2 & $\mathrm{TJ}-2$ & 1 & - & MUD & CQ-mQ (80\%), MQ (10\%), CAL-LF (5\%) & - & $\mathrm{MOL}, \mathrm{CaCO}_{3}, \mathrm{OF}$ & MAR-CS & 5 & $\begin{array}{l}1,2 \\
3\end{array}$ \\
\hline B1 & $\mathrm{J} 1-1$ & 1 & BRE & MUD & NOTS & NOTS & NOTS & MAR & 4 & 1,2 \\
\hline $\mathrm{C} 1$ & $\mathrm{~J} 2-1$ & 0 & LCR & MUD & CQ (70\%-90), mQ CAL-LF & FOR, ESP-T, BIV & $\mathrm{FEN}, \mathrm{CaCO}_{3}, \mathrm{Q}-\mathrm{TER}$ & MAR & 5 & 1,2 \\
\hline $\mathrm{C} 2$ & $\mathrm{~J} 2-1$ & $1,2,3,4$ & LCR & MUD & CQ $(90 \%), \mathrm{mQ}, \mathrm{CAL}-\mathrm{LF}$ & FOR, ESP-T, BIV & $\mathrm{FEN}, \mathrm{CaCO}_{3}, \mathrm{Q}-\mathrm{TER}$ & MAR & 6 & 1,2 \\
\hline C3 & $\mathrm{J} 2-2$ & 0 & LCR, PER & MUD & CQ-mQ (70\%), MQ CAL-LF (10\%) & ESP-M, FOR, GAS (?), INS & FEN, MOL, $\mathrm{OF}, \mathrm{CaCO}_{3}$ & MAR & 5 & 1,2 \\
\hline C3 & $\mathrm{J} 2-2$ & 1,2 & LCR, PER & MUD & CQ-mQ (70\%), MQ CAL-LF (10\%) & ESP-M, FOR, GAS (?), INS & FEN, MOL, $\mathrm{OF}, \mathrm{CaCO}_{3}$ & MAR & 5 & \\
\hline C3 & $\mathrm{J} 2-2$ & 4 & LCR & MUD & CQ-mQ (70\%), MQ CAL-LF (10\%) & ESP-M, FOR, GAS (?), INS & FEN, MOL, OF, $\mathrm{CaCO}_{3}$ & MAR & 5 & \\
\hline C3 & $\mathrm{J} 2-2$ & 0,1 & PER & WAC & CQ-mQ (65\%), MQ (5\%), CAL-LF (10\%) & $\begin{array}{l}\text { ESP-M (?), ESP-T, FOR, CHA-S, GAS, } \\
\text { INS (?) }\end{array}$ & FEN, MOL, OF, $\mathrm{CaCO}_{3}$, PEO, $\mathrm{MO}$ (?) & MAR. & 5 & 1,2 \\
\hline C4 & $\mathrm{J} 2-3$ & 0,1 & MLR, PER & MUD, WAC & CQ-mQ (75\%), MQ (5\%), CAL-LF (5\%) & ESP-M, FOR (?), GAS & FEN, $\mathrm{MOL}$ (?), $\mathrm{OF}, \mathrm{CaCO}_{3}, \mathrm{MO}$ (?) & MAR & 5 & 1,2 \\
\hline $\mathrm{C} 4$ & $\mathrm{~J} 2-3$ & $1,2,3,4$ & MLR, PER & MUD, WAC & CQ-mQ (75\%), MQ (5\%), CAL-LF (5\%) & ESP-M, FOR (?), GAS, OST & FEN, $\mathrm{MOL}$ (?), $\mathrm{OF}, \mathrm{CaCO}_{3}, \mathrm{MO}$ (?) & MAR & 5 & \\
\hline D1 & $\mathrm{J} 3-1$ & 0 & LCR & MUD & CQ-mQ (90\%), CAL-LF (<5\%), MQ (<5\%) & GAS & $\mathrm{CaCO}_{3}, \mathrm{OF}$ & $\begin{array}{l}\text { CONT-LAC/ } \\
\text { MAR }\end{array}$ & 5 & 1,2 \\
\hline D3 & $\mathrm{J} 3-1$ & 4 & LAM & MUD & CQ-mQ (90\%), CAL-LF (<5\%), MQ (<5\%) & CHA-O (?), CHA-S (?) & OF & $\begin{array}{l}\text { CONT-LAC/ } \\
\text { MAR }\end{array}$ & 8 & 1,2 \\
\hline D4 & $\mathrm{J} 3-2$ & 0 & LAM, PER & WAC, PAC & CQ-mQ (70\%), CAL-LF (5\%) & BIV, ESP-M, OST, GAS, CHA-S & $\mathrm{CaCO}_{3}, \mathrm{MOL}, \mathrm{OF}$ & $\begin{array}{l}\text { CONT-LAC/ } \\
\text { MAR }\end{array}$ & 6 & 1,2 \\
\hline D2 & $\mathrm{J} 3-3$ & $0,1,2,3$ & $\begin{array}{l}\text { PER, BRE, } \\
\text { LAM }\end{array}$ & MUD, GRN & $\begin{array}{l}\text { CQ-mQ (60-90\%), CAL-LS (10-5\%), MQ } \\
(<5 \%)\end{array}$ & GAS, CHA-S, CHA-O, BIV & $\begin{array}{l}\text { MOL, FEN, INT, PEO, OF, MO, CaCO3, Q- } \\
\text { TER }\end{array}$ & $\begin{array}{l}\text { CONT-LAC/ } \\
\text { MAR }\end{array}$ & 7 & 1,2 \\
\hline D3 & $\mathrm{J} 3-3$ & 4 & $\begin{array}{l}\text { PER, BRE, } \\
\text { LAM }\end{array}$ & WAC & CQ (55\%), mQ(30\%), MQ CAL-LF(5\%) & BIV & OF, TER & $\begin{array}{l}\text { CONT-LAC/ } \\
\text { MAR }\end{array}$ & 8 & 1,2 \\
\hline D5 & $\mathrm{J} 3-4$ & 0 & LCR, PER & PAC, GRN & CQ-mQ (50\%), mQ (5\%), CAL-LF (5\%) & ESP-T, FOR (?), GAS & $\begin{array}{l}\text { MOL, OF, PEO, Q-TER, MOS, } \mathrm{CaCO}_{3}, \mathrm{OO} \\
\text { (?) }\end{array}$ & $\begin{array}{l}\text { CONT-LAC/ } \\
\text { MAR }\end{array}$ & 5 & \\
\hline E1 & C2s-1 & 1 & LAM & MUD, PAC & mQ-cQ, CAL-LF, CAL-LS, OP & BIV, OST, CHA-S & PEO, PEL, OOI, Q-TER, OF & MAR & 5 & 1,2 \\
\hline E2 & C2s-2 & 0,1 & LAM & MUD, PAC & $\mathrm{CQ}(95 \%), \mathrm{mQ}$ CAL & FOR, ESP-M, BIV, OST, RAD & PEO, PEL, OOI?, Q-TER, OF & MAR & 6 & 1,2 \\
\hline E2 & C2s-2 & 4 & LAM & MUD, PAC & $\mathrm{CQ}, \mathrm{mQ}$ CAL-LF & FOR, ESP-M, BIV, OST, RAD & PEO, PEL, OOI?, Q-TER, OF & MAR & 6 & 1,2 \\
\hline E3 & $\mathrm{C} 2 \mathrm{~s}-3$ & 0,1 & LAM & MUD, PAC & CQ MQ CAL, OP & FOR, ESP-M, BIV & PEO, PEL, OOI?, Q-TER, MOS, OF & MAR & 6 & 1,2 \\
\hline $\mathrm{E} 4$ & $\mathrm{C} 2 \mathrm{~s}-4$ & 4 & LAM, PER & MUD, PAC & CQ MQ CAL-LF & OST, CHA-S, BIV & Q-TER & MAR & 7 & 1,2 \\
\hline E5 & C2s-5 & 1 & LAM, PER & MUD & CQ-mQ (70-90\%), CAL-LF & FOR, ESP-M, BIV, OST & $\mathrm{CaCO}_{3}$ & MAR & 6 & 1,2 \\
\hline E6 & C2s-6 & 4 & LAM, PER & MUD & CQ-mQ (90\%), MQ (<5\%), CAL (10\%) & FOR, ESP-M, BIV, OST & FEN, OF & MAR & 9 & 1,2 \\
\hline E7 & $\mathrm{C} 2 \mathrm{~s}-7$ & 4 & LAM & MUD & $\mathrm{CQ}, \mathrm{mQ}$ CAL & FOR, ESP-M, BIV, OST & NOTS & MAR & 8 & 1,2 \\
\hline F1 & ? & 3 & BRE & - & NOTS & NOTS & NOTS & CONT & 6 & \\
\hline F2 & O-MC-1 & 1 & - & MUD & CQ, CAL-LS, OP & NOTS & Q-TER & CONT & 6 & 1,2 \\
\hline G1 & Eo-1 & 0,1 & BRE & MUD & $\mathrm{OP}$ & NOTS & NOTS & CONT-LAC & 6 & 1,2 \\
\hline G2 & Eo-2 & 0,1 & BRE & MUD & CAL & NOTS & NOTS & CONT-LAC & 6 & 1,2 \\
\hline G3 & Eo-3 & 4 & BRE & MUD & CAL & NOTS & NOTS & CONT-LAC (?) & 6 & \\
\hline H1 & C-PE-1 & 0,1 & - & CNG, SAN & OP-CT, ALU, CAL-LF, KAO, SME & - & Q-TER, FEL, OF & CONT & 4 & 4 \\
\hline $\mathrm{H} 2$ & C-PE-2 & 0,1 & - & MUD-san & OP-CT, ALU, CAL-LF, KAO, SME & - & Q-TER, FEL & CONT & 6 & 4,5 \\
\hline H3 & C-P\&-2 & 0,1 & - & $\begin{array}{l}\text { SAN, MUD- } \\
\text { san }\end{array}$ & NOTS & NOTS & NOTS & CONT & 4 & \\
\hline I1 & MC-1 & $\begin{array}{l}0,1,2,3 \\
4\end{array}$ & & & & & & CONT-LAC & 6 & 1,2 \\
\hline I2 & MC-2 & 0,1 & - & MUD & $\mathrm{MQ}, \mathrm{CAL}$ & GAS, CHA-O & $\mathrm{OF}, \mathrm{CaCO}_{3}, \mathrm{MO}$ & CONT-LAC & 6 & \\
\hline $\mathrm{I} 2$ & MC-2 & $2,3,4$ & - & MUD & $\mathrm{MQ}, \mathrm{CAL}$ & GAS, CHA-O & - & CONT-LAC & 6 & \\
\hline I3 & MC-3 & 0,1 & BRE, LAM & WAC, PAC & $\mathrm{MQ}, \mathrm{mQ}, \mathrm{CAL}$ & CHA-S, CHA-O, GAS, & FEN, $\mathrm{CaCO}_{3}$, Q-TER & CONT-LAC & 6 & \\
\hline I3 & MC-3 & $2,3,4$ & BRE, LAM & WAC, PAC & $\mathrm{MQ} \mathrm{mQ}, \mathrm{CAL}$ & CHA-S, CHA-O, GAS, & - & CONT-LAC & 6 & \\
\hline
\end{tabular}




\section{CQ, CAL-LS}

CQ, MQ, CAL-LS, OP

$\begin{array}{ll}\text { MUD, WAC } & \text { CQ, mQ, CAL-LF, } \\ \text { MUD, WAC } & \text { MQ mQ, CAL-LF, CAL-LS }\end{array}$

MUD, WAC

MUD, WAC

$\begin{array}{ll}\text { MUD } & \text { OP-CT, SME } \\ \text { MUD } & \text { MQ OP-CT, SME }\end{array}$

$M Q, M Q, C A L-L F$

OP-CT, SME

- $\quad$ MQ CAL

MQ CAL

$\mathrm{Q}$

$\mathrm{MQ}$

CAL, $\mathrm{mQ}$
$\mathrm{MQ}$

MQ
OP

OP

Q

$\mathrm{mQ}$

MQ mQ
$-$

FEN, $\mathrm{CaCO}_{3} \mathrm{OF}, \mathrm{Q}$-TER

$\mathrm{CaCO}_{3}, \mathrm{OF}, \mathrm{Q}-\mathrm{TER}$

CONT-LAC

CONT-LAC

CONT (?)

CONT

NOTS

NOTS OF

NOTS

NOTS

OF, Q-TER (?)

Q-TER

NOTS

MOS

NOTS

OF

NOTS

NOTS

a Types follow geologic map convention code and a sequential number

b 0 (In situ outcrop), 1 (subprimary outcrops), 2 (colluvial gathering), 3 (recent river deposits), 4 (old alluvial deposits), and 5 (only observed in archaeological sample),

c BRE (brechoidal), LAM (lamination), LCR (liesegang concentric rings), MLR (multiple liesegang rings), PER (peloidal relict).

' CNG (conglomerate), GRN (grainstone), MUD (mudstone), MUD-san (sandy mudstone), PAC (packstone), SAN (sandstone), WAC (wackstone).

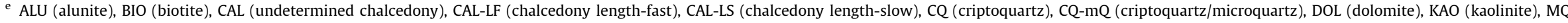
f

(bivalve), BRA (brachiopod), BRY (Bryozoa), CHA-O (Charophyta gyrogonite), CHA-S (Charophyta stem), CRI (crinoids), DIA (diatom), ECH (echinoid), ESP-M (monoaxone spicule), ESP-T (triaxone spicule), FOR (foraminifer), GAS (gastropod), INS (insertae sedis), OST (ostracod), RAD (radoliarian).

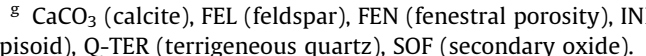

h CONT (continental), CONT-AL (alluvial), CONT-HYD (hydrothermal), CONT-Lac (lacustrine), MAR (marine), MAR-CS (marine continental shelf).
i

i Experimental evaluation of knapping quality $(1 / 10)$

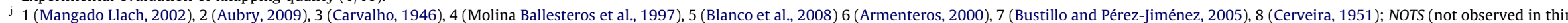
section) 
Table 3

Effectives of raw material categories defined in Table 2 used in the Côa Valley.

\begin{tabular}{|c|c|c|c|c|c|c|c|c|c|c|c|c|c|c|c|c|c|c|c|}
\hline \multirow[t]{3}{*}{ Raw material $^{\mathrm{a}}$} & & & \multicolumn{13}{|c|}{ Sites $^{\mathrm{b}}$} & \multicolumn{2}{|c|}{ Distance $^{c}$} & \multirow[t]{3}{*}{ Origin } & \multirow[t]{3}{*}{ Range } \\
\hline & & & \multirow{2}{*}{$\begin{array}{l}\text { OG4 } \\
3\end{array}$} & \multicolumn{2}{|c|}{ OG14 } & \multicolumn{6}{|c|}{ CAR } & \multirow{2}{*}{$\begin{array}{l}\text { INS } \\
2\end{array}$} & \multirow{2}{*}{$\begin{array}{l}\text { QBS } \\
3\end{array}$} & \multicolumn{2}{|l|}{ FAR } & \multirow[b]{2}{*}{$\mathrm{km}^{\mathrm{d}}$} & \multirow[b]{2}{*}{$\mathrm{hrs}^{\mathrm{e}}$} & & \\
\hline & & & & $2 c$ & 3 & 4.6 & 4.7 & 4.8 & 4.9 & 4.10 & 4.11 & & & 4 & $\begin{array}{l}9 / \\
10 \\
\end{array}$ & & & & \\
\hline \multirow{20}{*}{$\begin{array}{l}\text { Fine-grained } \\
\text { rocks }\end{array}$} & \multirow[t]{13}{*}{ Flint } & $\mathrm{A} 1 / 2$ & - & 2 & - & 1 & 5 & 42 & 2 & 8 & 2 & - & 1 & - & - & 130 & 21 & SW (Portuguese & \multirow{13}{*}{$\begin{array}{l}\text { Long- } \\
\text { distance }\end{array}$} \\
\hline & & $\mathrm{C} 1 / 4$ & 8 & 6 & 2 & 42 & 73 & 48 & 60 & 45 & 24 & 4 & 2 & - & 5 & 149 & 24 & Estremadura) & \\
\hline & & $\mathrm{D} 2 / 3$ & 31 & 17 & - & 4 & 5 & $4 \varepsilon$ & 8 & 16 & 9 & - & - & 3 & - & 180 & 30 & & \\
\hline & & $\mathrm{E} 2 / 7$ & 14 & 8 & 5 & 21 & 22 & 21 & 18 & 96 & 81 & 11 & 3 & 19 & - & 202 & 31 & & \\
\hline & & $\mathrm{F} 1$ & - & - & - & - & - & - & - & - & - & - & - & - & - & - & - & & \\
\hline & & F2 & - & - & - & - & - & - & - & - & - & - & - & - & - & - & - & & \\
\hline & & I1 & 144 & 16 & 16 & 41 & 85 & $59 \varepsilon$ & 89 & 402 & 331 & 15 & 6 & 10 & 2 & 212 & 23 & East (Northern Meseta) & \\
\hline & & $\mathrm{I} 2 / 3$ & 18 & 4 & 5 & 10 & 19 & 132 & 29 & 134 & 85 & 2 & 7 & - & - & 212 & 23 & & \\
\hline & & $\mathrm{I} 5 / 6$ & - & - & - & 1 & 1 & 42 & 2 & 32 & 9 & - & - & - & - & 268 & 26 & & \\
\hline & & 17 & 7 & 3 & 4 & 8 & 8 & 15 & 4 & 49 & 30 & 9 & 2 & - & - & 197 & 26 & & \\
\hline & & $\mathrm{I} 11$ & - & 1 & - & 3 & 1 & 42 & 2 & - & - & - & 2 & 4 & - & 159 & 24 & & \\
\hline & & $\begin{array}{l}\text { Not } \\
\text { determined }\end{array}$ & - & 2 & - & - & - & - & - & 96 & 62 & - & - & - & - & - & - & & \\
\hline & & Burnt & - & 2 & - & - & - & - & - & 20 & 81 & 2 & - & 7 & - & - & - & & \\
\hline & \multirow{10}{*}{$\begin{array}{l}\text { Hydrothermal } \\
\text { veins }\end{array}$} & $\mathrm{J} 3 / 4$ & - & - & - & - & - & - & - & 11 & 9 & 1 & - & 15 & - & 86 & 14 & SW & Intermediate \\
\hline & & $\mathrm{H} 1$ & 2 & - & - & 4 & 1 & 3 & 1 & 1 & - & - & - & - & - & 111 & 15 & $\mathrm{E}$ & \\
\hline & & $\mathrm{H} 2$ & 6 & 2 & - & 5 & 11 & $9 \varepsilon$ & 8 & 22 & 10 & - & - & - & 2 & 109 & 15 & & \\
\hline & & $\mathrm{J} 1$ & 21 & - & 8 & 12 & 26 & 24 & 14 & 11 & 12 & - & 32 & - & - & 38 & 7 & & Local \\
\hline & & $\mathrm{J} 2$ & 3 & - & - & 5 & 4 & 72 & 2 & 5 & - & 1 & 22 & 26 & - & 28 & 5 & & \\
\hline & & K1 & 26 & 16 & 5 & 2 & 5 & 8 & 3 & 7 & 3 & 2 & - & - & - & 15 & 3 & & \\
\hline & & M1 & 1 & 25 & 3 & - & - & - & - & 2 & 6 & 1 & 8 & 2 & - & 23 & 5 & & \\
\hline \multirow{4}{*}{$\begin{array}{l}\text { Coarse-grained } \\
\text { rocks }\end{array}$} & & Rhyolite & 19 & 19 & 1 & - & - & - & - & - & - & 3 & 3 & 2 & - & -1 & -1 & & \\
\hline & & Quartz & 7557 & 1316 & 25 & $x$ & $x$ & $x \quad \gamma$ & $x$ & 9817 & 5967 & 836 & 774 & 4974 & 195 & -1 & -1 & & \\
\hline & & Rock crystal & 968 & 92 & 108 & $x$ & $x$ & $x$ & $x$ & 5564 & 3411 & 142 & 16 & 237 & - & $\begin{array}{l}-1 / \\
25\end{array}$ & $\begin{array}{l}-1 / \\
25\end{array}$ & & \\
\hline & Meta-sediment & Quartzite & 971 & 71 & 174 & $x$ & $x$ & $x \quad \gamma$ & $x$ & 11875 & 8251 & 242 & 118 & 825 & 156 & -1 & -1 & & \\
\hline
\end{tabular}

\footnotetext{
a See Table 2 for raw material description.

b Olga Grande 4 (OG4), Olga Grande 14 (OG14), Cardina 1 (CAR), Ínsula (INS), Quinta da Barca Sul (QBS), Fariseu (FAR).

c Determined from the six sites' mean centre.

d Straight-line Euclidean distances.

e Cost distances using Tobler's (1993) hiking function (see text for details).

$x$ present but unrecorded.
}

closest source to the site. In most cases this choice was not complicated once most of the raw materials share the same limited coterminous regional distribution with the exceptions of type I11 and mostly type I1 (see Table 2). Type I11 corresponds to lacustrine Miocene flint available in primary position outcrops and in secondary position in the Tagus terraces, detected from El Puente de Arzobispo to Vila Velha de Ródão, along more than $200 \mathrm{~km}$ of the river basin. Type I1 is a ubiquitous siliceous type of rocks that can be found on both sides of the Iberian Central Mountain System, either on the Southern side at Rielves (Bustillo, 1976) or the Northern Plateau near Valladolid and Burgos (Armenteros, 2000; IGME, 1982). Once more, we have decided to use the closest source to the sites in calculating regional source use.

\section{Lithic raw material origins in the Côa Valley}

The Côa Valley data reveals the use of raw materials from four different areas of origin. Schist, granite, quartzite, quartz and rock crystal are available locally, in primary or secondary position in the areas surrounding each site, depending on local geology, but all within $1 \mathrm{~h}$ radius. Veins of fine-grained siliceous rocks (J1, J2, K1 and $\mathrm{M} 1$ ), are present in very circumscribed sources, corresponding to less than $7 \mathrm{~h}$ of walking. Walking away from the Côa Valley, certain raw material sources are located at about $14-15 \mathrm{~h}$, in two opposing directions, to the south-westwards (J4) and eastwards (H1 and H2). Finally, in these same directions, the most distant flint sources are located between 21 and $31 \mathrm{~h}$ walking (A1/2, C1/4, D2/3, E2/7 south-westwards; I1, I2/3, I5/6 and I7 eastwards) (Fig. 2).

The data obtained for the Côa Valley reveal that, during all Upper Palaeolithic occupation phases, lithic series are clearly dom- inated by local quartzite and quartz, but also include a small proportion of regional fine-grained siliceous rocks, as well as flint and other fine-grained siliceous rocks from distant sources (Fig. 2, Table 3). However, despite being suitable for knapping, some regional lithic raw materials detected to the north of the Douro valley were not used in any of the Upper Palaeolithic series studied (Fig. 2), including the regional sources of spatially localised Precambrian chert (L1), and volcanic opal (J6).

Overall, the occurrences of different local or regional raw materials used from the Gravettian to the Final Magdalenian in the Côa Valley, do not reveal significant differences in their sourcing. All the raw material sources, be they regional or distant, South-western or Eastern, continue to be exploited along the Upper Palaeolithic sequence in the Côa Valley.

However, one can detect a diachronic trend. Gravettian assemblages from Olga Grande 4 (Layer 3), Olga Grande 14 (Layer 3), Cardina 1 (Layers 4, 10 and 4,11), and Însula (Layer 2), have revealed a better representation of quartzite relatively to quartz, when compared to Final Magdalenian occupations, such as Fariseu and Quinta da Barca Sul assemblages, in spite of the local availability of both materials (Table 3 ).

In terms of regional and distant fine-grained sources, one also notes some differences. Generally, the same sources continue to be present throughout the entire sequence. However, during the Proto-Solutrean and Final Magdalenian, regional sources tend to overcome distant ones.

Cardina 1, which is the best example for detecting a diachronic trend due to the long stratigraphy and large assemblage, shows that the relative amount of exotic raw material from beyond $197 \mathrm{~km}$ (the median of the exotic raw material distances present; 
Table 4

Effectives of raw material categories defined in Table 2 used in the Portuguese Estremadura lithic assemblages studied.

\begin{tabular}{|c|c|c|c|c|c|c|c|c|c|c|c|c|c|c|c|c|c|c|c|c|c|c|c|c|c|}
\hline \multirow[t]{3}{*}{ Raw material ${ }^{a}$} & & & \multicolumn{23}{|c|}{ Sites $^{\mathrm{b}}$} \\
\hline & & & \multicolumn{2}{|l|}{ BG } & \multirow{2}{*}{$\begin{array}{l}\mathrm{BE} \\
2 \mathrm{ab}\end{array}$} & \multirow{2}{*}{$\begin{array}{l}\mathrm{BG} / \mathrm{BE} \\
\mathrm{km}^{\mathrm{c}}\end{array}$} & \multirow{2}{*}{$\begin{array}{l}\text { Dist } \\
\text { hrs }^{\mathrm{e}}\end{array}$} & \multirow{2}{*}{$\begin{array}{l}\mathrm{VB} \\
3 \mathrm{~b}, 3 / \\
4 \\
\end{array}$} & \multicolumn{7}{|l|}{$\mathrm{VC}$} & \multicolumn{2}{|c|}{ VB/VC Dist } & \multirow{2}{*}{$\begin{array}{l}\text { LCO } \\
3\end{array}$} & \multicolumn{2}{|c|}{ LCO Dist } & \multicolumn{3}{|l|}{ TMA } & \multicolumn{2}{|c|}{ TMA Dist $^{\mathrm{c}}$} \\
\hline & & & $\begin{array}{l}8 \mathrm{c} / \\
9 \mathrm{a}\end{array}$ & $9 \mathrm{~b}$ & & & & & 3 & 4 & 5 & 6 & $\begin{array}{l}6 / \\
7 \\
\end{array}$ & 7 & $8 \mathrm{~b}$ & $\mathrm{~km}^{\mathrm{c}}$ & $\mathrm{hrs}^{\mathrm{e}}$ & & $\mathrm{km}^{\mathrm{c}}$ & $\mathrm{hrs}^{\mathrm{e}}$ & $2 s 1$ & $2 \mathrm{~s} 2$ & Total $^{\mathrm{d}}$ & $\mathrm{km}^{\mathrm{c}}$ & $\mathrm{hrs}^{\mathrm{e}}$ \\
\hline \multirow[t]{21}{*}{ Fine-grained rocks } & Flint & $\mathrm{A} 1 / 2$ & - & - & - & - & - & - & - & - & - & - & - & - & - & - & - & - & - & - & - & - & - & - & - \\
\hline & & $\mathrm{C} 1 / 2$ & 4 & - & - & 36 & 4 & 7 & 32 & 59 & 55 & 9 & 1 & 4 & - & 29 & 4 & - & - & - & - & - & - & - & - \\
\hline & & $\mathrm{C} 3 / 4$ & 83 & 95 & 14 & 3 & -1 & $x$ & 511 & 828 & 648 & 679 & 27 & 826 & 190 & 1 & -1 & 7 & $\begin{array}{l}-1 / \\
11\end{array}$ & $\begin{array}{l}-1 / \\
3\end{array}$ & 1 & 1 & 5 & 29 & 7 \\
\hline & & D2/3 & 4 & 10 & 3 & 28 & 5 & 24 & 15 & 37 & 29 & 39 & 1 & 39 & 8 & 34 & 5 & 452 & 25 & 5 & 2 & - & 10 & 60 & 13 \\
\hline & & E2/7 & 20 & 32 & 6 & 35 & 5 & 54 & 100 & 261 & 303 & 367 & 16 & 432 & 6 & 44 & 5 & 3120 & 12 & 3 & 1310 & 844 & 9129 & 2 & -1 \\
\hline & & $\mathrm{F} 1$ & - & - & - & - & - & - & - & - & - & - & - & - & - & - & - & 73 & 12 & 3 & 40 & 11 & 205 & 2 & -1 \\
\hline & & F2 & - & 3 & - & 23 & 3 & 1 & 6 & 4 & 4 & 1 & - & - & - & 25 & 4 & - & - & - & - & - & - & - & - \\
\hline & & I1 & - & - & - & - & - & - & - & - & - & - & - & - & - & - & - & - & - & - & - & - & - & - & - \\
\hline & & $12 / 4$ & - & - & - & - & - & - & - & - & - & - & - & - & - & - & - & - & - & - & - & - & - & - & - \\
\hline & & $15 / 6$ & - & - & - & - & - & - & - & - & - & - & - & - & - & - & - & - & - & - & - & - & - & - & - \\
\hline & & 17 & - & - & - & - & - & - & - & - & - & - & - & - & - & - & - & - & - & - & - & - & - & - & - \\
\hline & & I11 & - & - & - & - & - & - & - & - & - & - & - & - & - & - & - & - & - & - & - & - & - & - & - \\
\hline & & $\begin{array}{l}\text { Not } \\
\text { determined }\end{array}$ & 5 & - & - & - & - & - & - & - & - & - & - & - & - & - & - & 280 & - & - & - & - & - & - & - \\
\hline & & Burnt & - & - & - & - & - & - & - & - & - & - & - & - & - & - & - & - & - & - & - & - & - & - & - \\
\hline & Hydrothermal & $\mathrm{J} 3 / 4$ & - & - & - & - & - & - & - & - & - & - & - & - & - & - & - & - & - & - & - & - & - & - & - \\
\hline & veins & $\mathrm{H} 1$ & - & - & - & - & - & - & - & - & - & - & - & - & - & - & - & - & - & - & - & - & - & - & - \\
\hline & & $\mathrm{H} 2$ & - & - & - & - & - & - & - & - & - & - & - & - & - & - & - & - & - & - & - & - & - & - & - \\
\hline & & $\mathrm{J} 1$ & - & - & - & - & - & - & - & - & - & - & - & - & - & - & - & - & - & - & - & - & - & - & - \\
\hline & & $\mathrm{J} 2$ & - & - & - & - & - & - & - & - & - & - & - & - & - & - & - & - & - & - & - & - & - & - & - \\
\hline & & K1 & - & - & - & - & - & - & - & - & - & - & - & - & - & - & - & - & - & - & - & - & - & - & - \\
\hline & & M1 & - & - & - & - & - & - & - & - & - & - & - & - & - & - & - & - & - & - & - & - & - & - & - \\
\hline \multirow{4}{*}{$\begin{array}{l}\text { Coarse-grained } \\
\text { rocks }\end{array}$} & & Rhyolite & - & - & - & - & - & - & - & - & - & - & - & - & - & - & - & - & - & - & - & - & & - & - \\
\hline & & Quartz & $x$ & $x$ & 48 & 3 & -1 & 125 & 213 & 394 & 259 & 327 & 21 & 497 & 4 & 4 & -1 & $x$ & 1 & -1 & 844 & 967 & 5998 & 2 & \\
\hline & & Rock crystal & - & - & - & - & - & - & - & 2 & - & - & - & - & - & $?$ & $?$ & - & - & - & $x$ & $x$ & $x$ & - & - \\
\hline & Meta-sediment & Quartzite & - & $x$ & 5 & 3 & -1 & 73 & 40 & 138 & 86 & 194 & - & 202 & 3 & 4 & -1 & $x$ & 1 & -1 & 156 & 391 & 1139 & 2 & \\
\hline
\end{tabular}

a See Table 2 for raw material description.

Buraca Grande (BG), Buraca Escura (BE), Vale das Buracas (VB), Vale dos Covões (VC), Lapa dos Coelhos (LCO), Terra do Manuel (TMA).

C Straight-line Euclidean distances.

e Cost distances using Tobler's (1993) hiking function (see text for details).

$x$ present but unrecorded. 


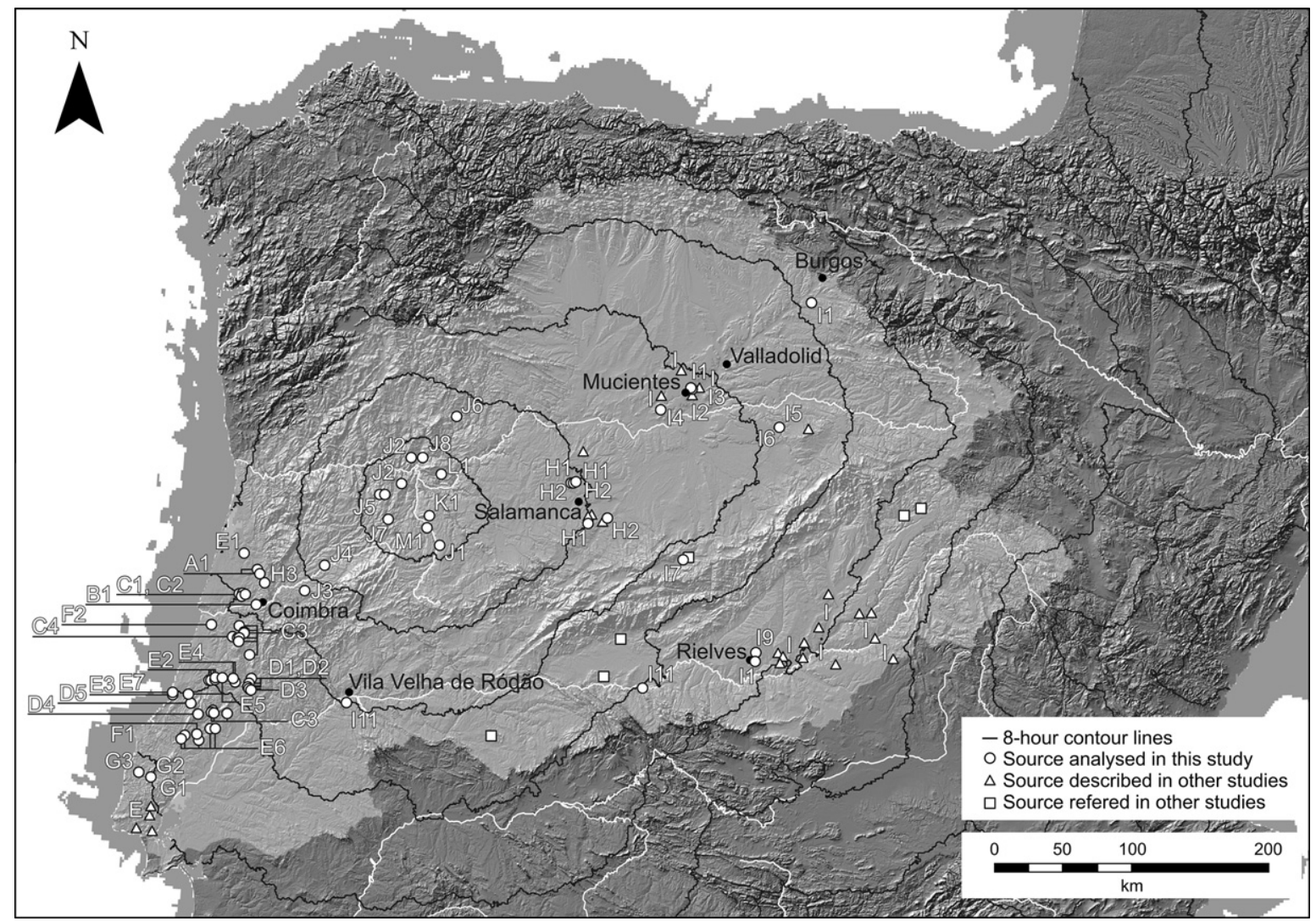

Fig. 2. Siliceous raw materials available between the Douro and Tagus basins with time costs estimated from a mean centre in the Côa Valley area.

Table 3) decreases trough time in the site sequence, as opposed to the raw materials below that distance.

In terms of the two geographic areas, after LGM, the ratio of Eastern sources decreases in favour of South-western flint (Fig. 3).

\section{Differential raw material use}

As noted earlier, all the lithic assemblages have been studied technologically and selected bladelet and flake tools, as well as non-retouched blanks, were submitted to use-wear analysis (Aubry and Araújo Igreja, 2009). Each piece was classified into a technological scheme: from block, nodule or pebble, to retouched flakes and tools, in order to establish the production processes for each different lithological group, their techniques and purposes (Fig. 4).

Our interpretation of the different production processes, hammer types and techniques (Pelegrin, 2000) used to remove blanks in the Côa Valley series has resulted in an ascription to seven categories. Each sequence was evaluated in terms of its effective representation at each site and layer. An example of technological description by raw material is presented in Fig. 4. Gravettian assemblages reveal quite complete local quartzite pebble reduction sequences, which are basically represented by the production of flakes. These short and thick flakes were probably used un-retouched or slightly retouched as end-scrapers and side-scrapers (Aubry and Araújo Igreja, 2009). Some pebbles, blocks and cores from such productions were also used as heat accumulators on fire-pits, either in the form of pebbles or discarded cores.

Throughout the Côa Upper Palaeolithic, quartz reduction sequences show the greatest diversity in technical processes of production, which is probably related to the variety of petrographic characteristics (crystal, fine grain to coarse quartz, milky to translucent), in relation to the origin of the material and the type of blocks used (pebbles, vein fragments). In the oldest Upper Palaeolithic occupation of the region attested in the layers 3 of Olga Grande 4 and 14 the morphology of blanks and numerous refitting reveals that fragments of local large vein quartz were used to produce thick flakes. At Cardina 1, a large range of quartz materials was used, revealing several reduction sequences to produce flakes and bladelets. At this site, the module of the scrapers made on milky quartz blanks, when compared to by-products of the quartz series, clearly indicates that the blanks were not produced in the excavated area. During the Final Magdalenian, at Fariseu, technological analysis has revealed the same situation: retouched tools made on cortical flakes produced from large quartz pebbles (more than $10 \mathrm{~cm}$ of diameter) were not produced on site (Gameiro et al., 2008).

With some exceptions in the numerically small series, the third most common raw material category is rock crystal (Table 3 ). Translucent rock crystals (from 1 to $3 \mathrm{~cm}$ in diameter) were available locally or regionally, while exceptionally large specimens ( $>5 \mathrm{~cm}$ in diameter) and smoked varieties associated to uranium mineralisation are present as veins in local schist and granite bedrock. They were used as blanks for bladelet and small flake production, with two distinct operative schemes (Fig. 4). The most common operative scheme is based on anvil bipolar percussion, applied directly on the crystal or on a flake, recognised by the stigmata observed on the products and cores. The second one, less represented, depends on an unidirectional production sequence, where bladelets are removed either by soft hammer direct percussion, using the longer axis and the intersection of the natural faces of the rock crystals, or by burin-type cores. Experimentation with this reduction sequence revealed that the anvil bipolar percussion reduction sequence, when applied on prepared flakes, allows the production of bladelets and a more intensive exploration of volumes smaller than $1 \mathrm{~cm}$ (Klaric et al., 2009). Based on the technological data available, the possible function of these pieces as intermediary pieces ("pièces esquillées") (Le Brun Ricalens, 2006) 
cannot be entirely excluded. However, the reduced module, the morphology and the exclusive use of fine-grained raw material to produce them, argue in favour of the interpretation of these pieces as cores for bladelet and small flake production, and not as intermediary tools.

Singularly, at Olga Grande 4, rock crystal is the raw material preferentially used for on-site production of blanks for microgravettes and retouched bladelets, by direct soft hammer removal, as well as bladelet and small flake productions, attested by numerous by-products probably used without transformation as cutting edge elements of composite projectile points (Fig. 4).

The next category of raw materials by number is composed of several regional fine-grained vein quartz and exogenous flint categories discarded at all the sites ( $\sim 5 \%$ of the total raw material used, on average). The technological study reveals the use of these raw materials to produce blanks for retouched bladelets, following the same reduction sequence as described for rock crystal. However, part of the regional fine-grained siliceous rocks arrived on site in the form of blocks or prepared cores (Fig. 4), differently from the exogenous flints, which show reduction sequences that correspond essentially to the final phases of bladelet production and the reuse of blanks, flakes or blade-tools (Fig. 4). We could establish some differences between flint productions. In the Gravettian layers of the granitic plateau sites (Olga Grande 4 and 14), they correspond basically to fragments of retouched bladelets, showing diagnostic fractures due to their use as projectiles and damaged tools (e.g., notches, scrapers), discarded or reused as blanks for the preparation of cores by bipolar percussion on anvil. At Cardina 1, a site located in the valley bottom, the Gravettian layers $(4,10$ and 4,11) present extremely reduced cores and core preparation and rejuvenation flakes, like tablets, indicating the on-site production of bladelets by soft hammer percussion on prismatic cores. However, the production of bladelets is essentially related to anvil bipolar percussion on flakes, or on small, fragmented retouched blades. These blades, retouched or not, were introduced in the Côa Valley as part of the tool-kit, and were reused after breakage.

During the Final Magdalenian, at Quinta da Barca Sul and Fariseu, the curved backed points and retouched bladelets were mainly

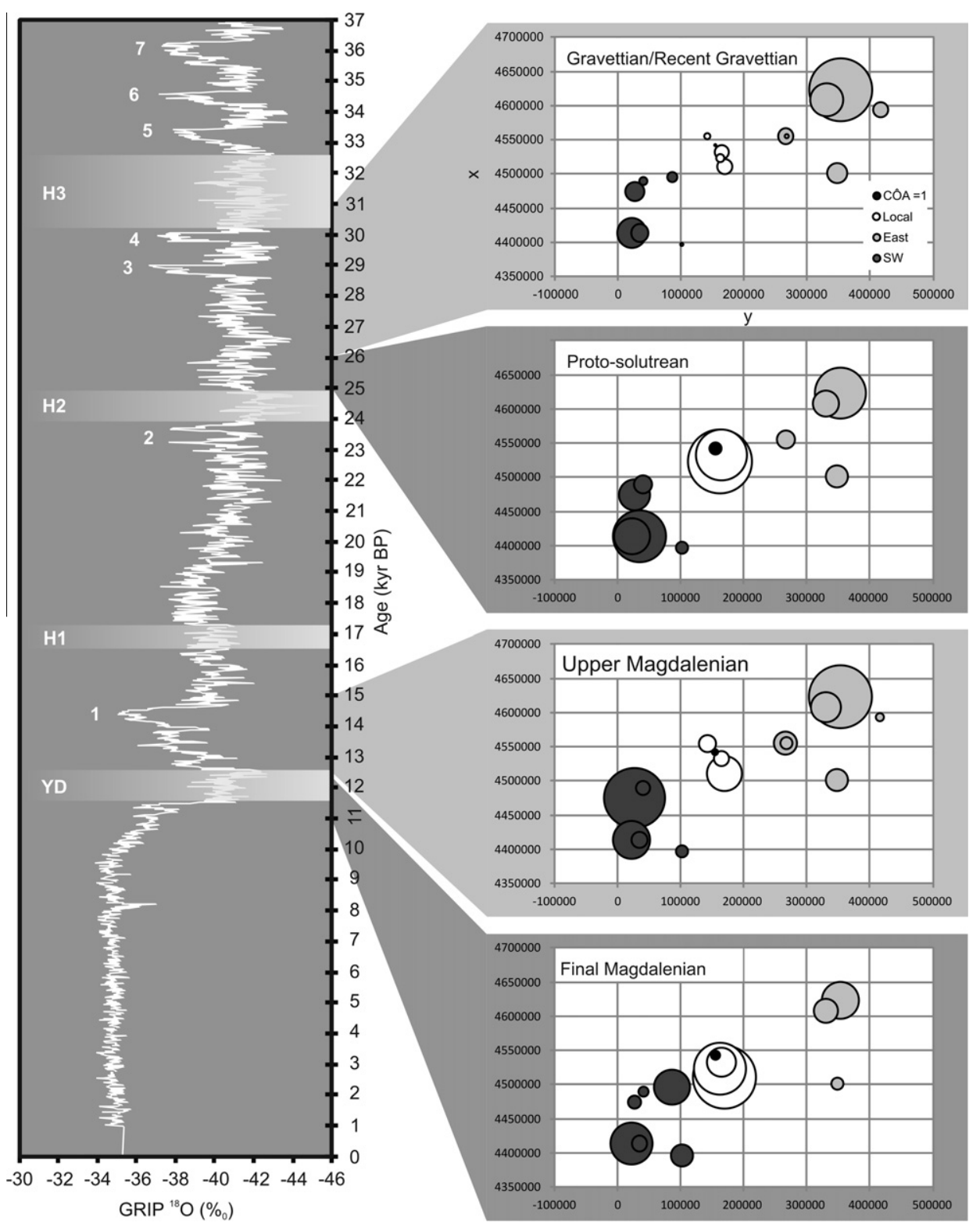

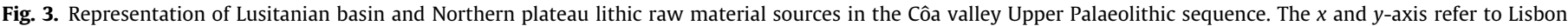
Hayford Gauss IGeoE coordinates, and relative abundance is indicated by the size of the circles. For scale purposes the Côa Valley circle size equals to 1. 


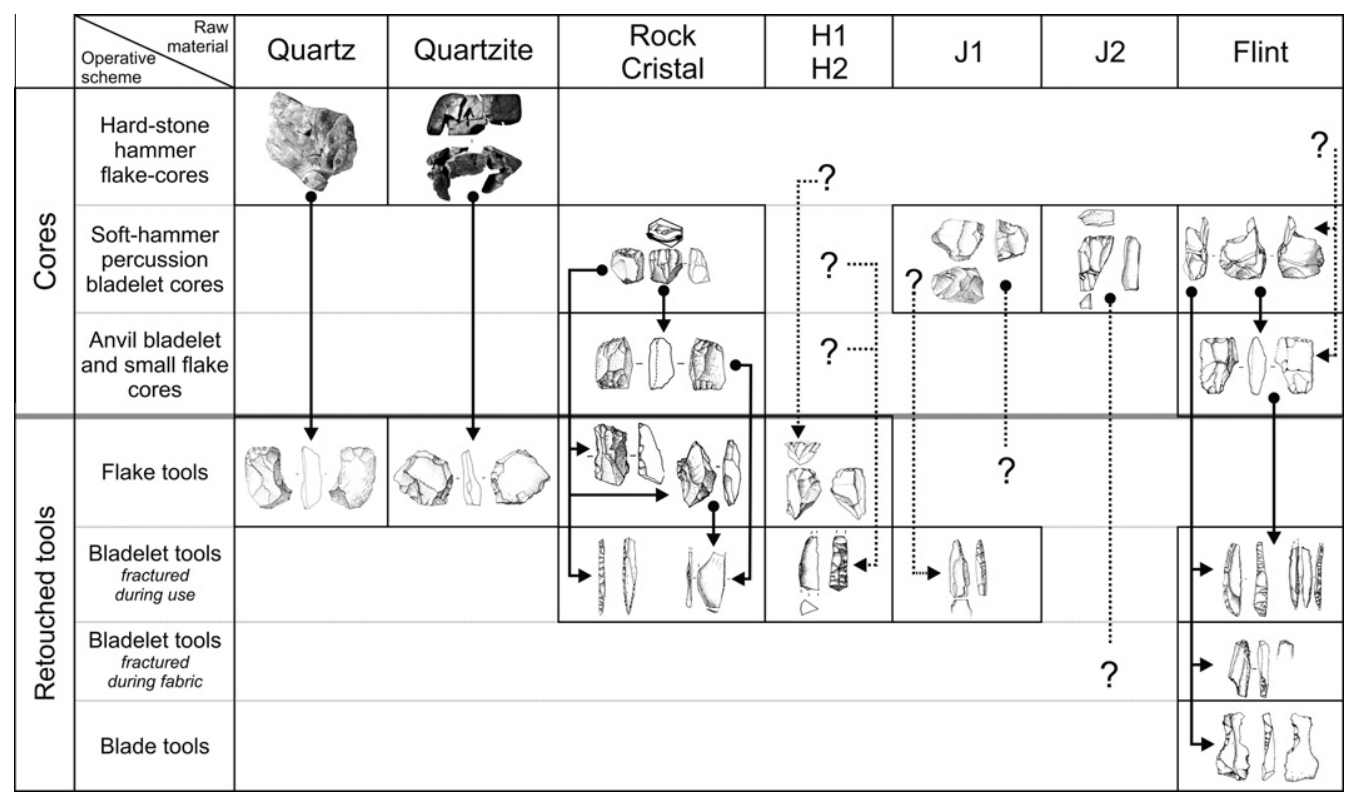

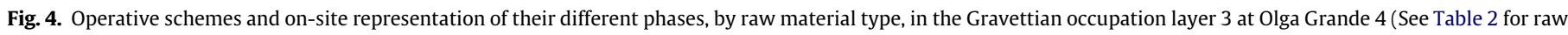
material categories description).

produced from regional fine grain siliceous vein rocks and some southern flint sources (Gameiro et al., 2008). The lithological diversity observed for the local and non local raw materials of the retouched bladelet tools and the identification of typical fractures suggest discard after use as projectile implements, which were replaced by tools made from regional fine-grained siliceous sources.

Despite these differences in the operative scheme of local and long-distance raw materials, no noticeable technological distinction can be made between materials coming from the East and the Southwest, namely in the Gravettian layers of Cardina 1 and Olga Grande 4, where these materials are numerically important and statistically relevant.

\section{Comparison with Upper Palaeolithic raw material procurement in Central Portugal (Sicó Massif and Serra d'Aire e Candeeiros)}

Of the six Upper Palaeolithic archaeological sites considered for comparison, four are located south of Coimbra, in the northern sector of the Meso-Cenozoic deposits of the Western Iberia margin (Wilson et al., 1989) (Fig. 1c and Table 1), including marine, littoral and continental sediments, often carbonates, covering the Iberian Hercynian crystalline basement (Ribeiro et al., 1979). Since 1991, a research project allowed us to recognise Middle and Upper Palaeolithic lithic assemblages preserved in occupation layers of caves and rock-shelters formed in the Middle Jurassic geological formations of the Sicó Massif (Aubry et al., 1994, 1997, 2001, 2008, 2010; Almeida et al., 2003), a geological formation bearing poor quality flint nodules (Aubry et al., 2001; Aubry, 2009). This limestone massif rises to less than $600 \mathrm{~m}$ above sea level and is presently located $40 \mathrm{~km}$ east of the Atlantic (Cunha, 1990).

The sites considered (Buraca Escura, Buraca Grande, Vale das Buracas and Abrigo do Vale do Covões) are caves or rock-shelters located in two fluviokarstic canyons cut in the Middle Jurassic carbonate formations of the western belt of the Sicó Massif (Cunha, 1990), all yielding flint nodules in limestone outcrops or in continental Cretaceous and Cenozoic deposits covering depression in the bedrock. The cultural attribution of each archaeological assemblage is based on typological evidence, ages obtained by absolute dating, palaeoenvironmental conditions inferred from the features of sediment and soil formation and its disconformities. This data allow us to propose a correlative framework (Table 1) for the stratigraphical and archaeological sequence of these four sites preserving Middle and Upper Palaeolithic (Gravettian, Solutrean and Magdalenian) occupation levels (Aubry et al., 2010).

The archaeological assemblages of the Sicó massif sites were analysed using the same protocol applied to the Côa Valley series, including systematic survey for locally available primary and secondary sources of lithic raw materials and their comparison with the archaeological record, completed by technological and use-wear analysis on a sample of retouched tools and blanks (Aubry et al., 2001, 2008; Gameiro et al., 2008; Aubry and Araújo Igreja, 2009).

Raw material procurement patterns revealed the use of the local quartz and quartzite pebbles, in all the series, despite the prevalence of a local poor quality flint, available at less than $1 \mathrm{~h}$ of walking distance. The data also show a systematic use of exogenous fine-grained flint from Upper Cretaceous (E2/7) and Upper Jurassic (D2/3) sources available at less than a $8 \mathrm{~h}$ walk distance southwards (Aubry et al., 2001; Gameiro et al., 2008) (Table 4). Technological study reveals that these fine-grained flint categories, having better qualities for knapping and for use in projectile technology than the local Middle Jurassic flint, were preferentially used to produce retouched bladelets. Other tool types were made on byproducts of the bladelet production sequence on prismatic or burin-cores, or via independent flake production schemes.

The two other sites used for comparison with the Côa Valley series are also located in the Portuguese Estremadura, or Lusitanian basin, towards the South, in the Serra dos Candeeiros area. Terra do Manuel is an open-air site, firstly excavated in the 1940s and continued in the 1990s (Zilhão, 1997), which identified two Recent Gravettian occupation levels ( 2 s1 and 2 s2), very similar to Cardina $1(4,10$ and 4,11$)$, confirmed by salvage excavation in the 2000s (Almeida et al., 2010).

This site is located very close to several major Cenomanian secondary flint sources in the Rio Maior region. Like the Sicó sites, Terra do Manuel occupations rely mainly on local flint (less than $1 \mathrm{~h}$ ). The raw material types are roughly the same as the ones used in the Sicó sites, however, considerable effort was made to obtain the Upper Jurassic flint (D2/3, 13 h) (Table 4).

Two very different typo-technological systems could be identified. The lower level presents a small number of flint cores when compared with the amount of flint debris, as opposed to quartz and quartzite, where pebble cores and flakes are present. These 
flakes were used differently according to raw material, quartz was retouched and quartzite used unretouched. The absent flint cores were probably prepared on-site for the production of backed and truncated bladelets, partially explored and then exported. In the upper level non-retouched bladelets and the other bladelet forms were produced from prismatic and thick carinated scraper cores.

Finally, Lapa dos Coelhos is a small cave open in the lower portion of the Almonda karstic system (Angelucci and Zilhão, 2009), which was excavated between 1995 and 2007 under the direction of F. Almeida (Almeida et al., 2004). The archaeological sequence, beginning during the Middle Palaeolithic, comprises Solutrean and Upper Magdalenian occupations (Almeida et al., 2004). The lithic assemblage from layer 3 (Table 1) was studied by Gameiro from a lithological and technical perspective (Gameiro et al., 2008). Raw material sources used in this site are generally the same as Terra do Manuel's, with the advantage of being closer to Middle (C3/4) and Upper Jurassic (D2/3) sources (all within $5 \mathrm{~h}$ walks) (Table 4). The different flint sources were used for the onsite production of small convergent bladelets extracted from prismatic, thick-scraper and burin cores (Gameiro et al., 2008). The blanks for thumbnail and on-flake scrapers consist of sub-products of this bladelet tool production. Quartz and quartzite pebbles were used to produce short flakes following a unidirectional or opposed platform reduction scheme.

\section{Discussion}

\section{Palaeoenvironmental forcing}

When interpreting the data presented above, a first variable to consider is the impact of climatic variation in ecosystems and resource distribution and availability just before and after the LGM.

Global climatic fluctuations during the Upper Palaeolithic sequence are well-known from the data established on the Portuguese continental margin (Lebreiro et al., 1995; Zahn et al., 1997; Bard et al., 2000; Shackleton et al., 2000; Thouveny et al., 2000; de Abreu et al., 2003; Skinner and Elderfield, 2007). The study of the last glacial period's record revealed various events of abrupt change associated with severe changes in surface water temperatures $\left(\sim 7^{\circ} \mathrm{C}\right.$ in decades; de Abreu et al., 2003) and a periodicity of 1500 years (Bond et al., 1997; Grootes and Stuiver, 1997; Debret et al., 2007). Several stadial/intestadial cycles have resulted in massive ice-discharges from the Northern Hemisphere ice sheets (Heinrich, 1988), every 7000-10,000 years, known as Heinrich Events (Bond et al., 1993), that have been described in cores along the Portuguese continental margin (Lebreiro et al., 1995; Cayre et al., 1999; Sánchez Goñi et al., 2000; Roucoux et al., 2001; d'Errico and Sánchez Goñi, 2003; de Abreu et al., 2003; Roucoux et al., 2005; Sánchez Goñi et al., 2008).

From deep-sea pollen records and forest macroremains occurring along the NW Iberia coast in northern Portugal, Zilhão (1997) has proposed a general picture for the Portuguese Estremadura where scot pine forest is mixed with open herbaceous scrublands and patches of temperate deciduous and Mediterranean forest. At the time of Heinrich Events, correlations between marine and continental proxy data evidence a large development of dry climate-type vegetation in western Iberia, with low percentages of arboreal pollen during cold events and herb and shrub vegetation with steppe species dominating. Lowered temperatures and precipitation were also accompanied by intensified winds leading to increased upwelling (Combourieu Nebout et al., 2002; Sánchez Goñi et al., 2002; Turon et al., 2003).

In central Portugal, faunal remains of Upper Palaeolithic age are known from cave and rock-shelter sites only, and data concerning Late Pleistocene fauna are scarce for all of the Northern Meseta. As a result of acid soils formed on metasediments and granites of the Côa Valley, as stated earlier, this kind of record is only available for layer 4 of the Fariseu site, an occupation dated by TL, OSL and ${ }^{14} \mathrm{C}$ AMS between 11 and 12,000 BP and assigned to the Younger Dryas (Aubry et al., 2010). Wild boar (Sus scrofa), red deer (Cervus elaphus), chamois (Rupicapra rupicapra), rabbit (Oryctolagus cuniculus), squirrel (Sciurus vulgaris), undetermined cyprinid and shad (Alosa alosa) were identified (Gabriel and Béarez, 2009). Comparison with species presence/absence and dating of layers I-VI of Peña de Estebanvela ( $\sim 11$ to $14,000{ }^{14} \mathrm{C}$ years BP) (Cacho Quesada et al., 2007), a sequence preserved in a rock-shelter located at the eastern limit of the Northern Meseta (Fig. 1a), reveals the same large mammal species, along with Equidae (Equus caballus and hydruntinus) and few carnivore remains (Felix silvestres, Lynx pardina, Canis lupus and Vulpes vulpes), with rabbit being the most represented species in all layers, despite a better representation of the Equidae remains in layers III and IV.

The faunal record for the Upper Palaeolithic sequence preserved in caves and rock-shelters of Central Portugal is slightly better known (Zilhão, 1997; Aubry, 2001; Davis, 2002; Hockett and Haws, 2009; Haws, 2011). Studies have revealed that rabbit remains show intentional cut marks and breakage patterns (Hockett and Bicho, 2000) and that the ratio of rabbit to ungulate increases from the Mousterian to the Magdalenian (Davis, 2002), which is explained as the result of a gradual increase of hunting pressure on the environment, probably due to higher human density. Red deer and wild boar dominate during the Lateglacial as opposed to a better representation of Equidae, ibex, chamois and aurochs before and during the LGM (Zilhão, 1997; Davis, 2002; Aubry et al., 2001; Haws, 2011). Thus, both in the Meseta and along the Atlantic coastline, the variation in animal resources reveals the same ecological trends from the end of the LGM to the Lateglacial.

Apparently, biotic resource forcing cannot be directly correlated with changes in the procurement of stone tool raw material over time, since it does not reveal clearly different origins from the Gravettian to Final Magdalenian/Azilian assemblages (Fig. 3). In the Côa Valley sites, local raw materials tend to gain importance over long-distance ones during the Proto-solutrean and the Final Magdalenian/Azilian (Fig. 3) and the predominance of Meseta sources over those from Portuguese Estremadura gradually decreases. However, all the raw material sources (local, eastern or south-western) were used, which, despite differences over time in their relative importance, implies the existence of a similar spatial organisation and social network throughout the Upper Palaeolithic. In the current state of knowledge, no correlation can be clearly established between palaeoenvironment and changes in the procurement of raw materials (Fig. 3), so other explanations must be found.

\section{Functional forcing}

Functional and technological arguments have been advanced as explanation for the presence of exotics in Central Australian sites, where they present more efficient edge-holding properties (Gould and Saggers, 1985).

In the Upper Palaeolithic of the Côa Valley, rock crystal, regional fine-grained vein quartz sources and long-distance flint sources were used to produce the same types of retouched bladelets in all the assemblages and with similar technological solutions (anvil bipolar percussion, associated with prismatic core reduction) (Fig. 4). Other raw materials, which were available locally, less than $50 \mathrm{~km}$ away, were used to produce the same specialised tools made on flint coming from distant sources. This fact is a strong argument against a strictly functional interpretation of the use of flint, independently of cultural factors. 
Comparison between Recent Gravettian reduction sequences from Cardina 1 and Terra do Manuel reveals that the production of the same truncated retouched bladelet on flint are the result of different reduction sequences (Klaric et al., 2009). This flexibility is interpreted as an adaptation to low resource availability. The anvil bipolar percussion used in the Côa Valley, away from flint sources, allows maximal exploitation of core volumes (Aubry, 2009). Terra do Manuel, close to large Cenomanian flint sources, features classical unipolar prismatic bladelet cores, as is also seen at the same time in sedimentary basin sites of Southern France (Klaric et al., 2009).

In the logistical Gravettian occupation of Olga Grande 4, most of the bladelet tools produced on-site are on rock crystal, although onsite reduction of exotic flints is also documented (Fig. 4). This fact strongly suggests that such exotic raw materials were not indispensable for this specific type of tool production. Two different Recent Gravettian layers from Cardina 1 (layers 4, 10 and 4,11) reveal the same general pattern in the ratio between the total of the finegrained remains from different sources and the proportion of these sources in bladelet tools (Fig. 5). With few exceptions, namely I1, part of the bladelet tools arrived as retouched bladelets, integrated or not in composite hunting tools, while a few nodule volumes, tools or flakes were introduced alongside and re-used on-site to produce bladelets or small flakes by anvil percussion (Fig. 4).

By itself, technology does not clearly explain the context of exotic raw material discard in the area. It is clear that some of the exotic raw materials, but also regional ones, arrived at the sites as blanks or finished products throughout the Upper Palaeolithic sequence. Even though flint is always present in the Côa Valley, its reduction sequence is one adapted to scarcity, having a clearly distinguishable chaîne opératoire from contemporary and similar tools in areas with abundance of this type of raw material.

This data proves that flint was not indispensable for the production of tools, since local raw materials were also used. However, it was used because it was better suited for knapping, more efficient for use without curation, and more appropriate for manufacturing the lithic components of hunting tools.

\section{Sociocultural models for the Côa Valley Upper Palaeolithic data}

Theoretically, the Côa Valley lithic assemblages could be the result of the gradual accumulation of remains discarded by distinct cultural groups, originating from the Northern Meseta and the Portuguese Estremadura, during successive, unrelated occupations that exploited the same local biotic resources (Bailey, 2007; Hertell and Tallavaara, 2011). The successive reutilization of the exact same open-air sites by distinct human groups is highly improbable and is refuted by the archaeological data from two logistical hunting occupations on the granitic plateau. Refitting of stones used to structure large fire pits and between knapped lithic debris in the Gravettian occupations of Olga Grande 4 and 14 shows that the Eastern and South-western raw material sources of bladelet tools present the same spatial patterns and were both used and discarded during short, specialised hunting occupations (Aubry, 2009; Fig. 6). Thus, the occupation of the same area on separated moments by different human groups from distinct regions cannot be advanced as an explanation for the origins and diversity of stone tool raw material sources used on-site.

Classically, other explanations of hunter-gatherer lithic raw material procurement involve two apparently colliding positions. One considers that raw material procurement is essentially embedded in daily subsistence activities (Binford, 1979), while the other argues, based on the long-distance displacement observed among present-day hunter-gatherer groups (Féblot-Augustins and Perlès, 1992), that some raw materials can also be interpreted as direct procurement from a site to one or more specific sources (Gould and Saggers, 1985), or the exchange of raw materials or tools between groups (Gamble, 1999).

Lithic raw materials, and especially so the fine-grained siliceous rocks, are not evenly distributed across the landscape and, as it depends on specific geological variables, the location of certain kinds of lithic raw materials may not coincide with that of other resources used by foragers. A number of ethnographic and archaeological examples show that raw materials can be introduced from distant sources. This fact can be explained by a utilitarian reason, as stated previously, but also by a broader adaptative reason. The presence of these exotic raw materials could be seen as evidence for the creation and maintenance of long-distance social networks. These social networks meet the regular needs of widely scattered groups (resources, partners) and can also function as a "safety net" in exceptional situations of resource scarcity (Gould and Saggers, 1985; Whallon, 2006). These exchange networks can be perceived as adaptive strategies, but they function also as cultural and ideological markers. Even though this type of exchange might be perceived as the opposite of embedded procurement, since it involves resources outside the landscape of habit or the strict home range, object transfers, namely raw materials, are in fact embedded in the activities groups perform (Polanyi, 1957; Gamble, 1999).

Thus, a variety of theoretical possibilities could account for the reality identified in the Côa Valley archaeological record (Fig. 7). In a first interpretative model, toolstone sources located in the Portuguese Estremadura, the Meseta regions and intermediary regions would correspond to parts of a large territory exploited at different times by the same human group in what could be defined as embeddedness in a foraging system (Binford, 1980) (Fig. 7a). A different scenario would be embeddedness in a collector/logistical system (Binford, 1980) or a pattern of concentration and dispersion (Lee and Daly, 1999), where groups are seasonally reunited in one aggregation area, after being separated for most of the year (Fig. 7b).

Compilation of ethnological literature of the annual land-use range of present-day hunter-gatherers (Kelly, 1995: Table 4-1) reveals that only the extreme case of subarctic Baffinland Inuit approaches the maximum area defined by the procurement of lithic raw materials discarded on each occupation of the Côa Valley (over $27,000 \mathrm{~km}^{2}$ in its maximal extension) (Fig. 8b). Except for equestrian (Crow Apsáalooke) and maritime cases, there are no hunter-gatherers known to cover an annual territory of such magnitude (Ellis, 2011). However, such extent can be observed in long-term mobility or lifetime ranges, notably among the Nunamiut (Binford, 1983) and the Montagnais (Hertell and Tallavaara, 2011). The areas defined by regional fine-grained siliceous rocks, in the Côa sites and for non local flint of the Portuguese Estremadura sites fall, however, within the limits of the annual land-use range of this type of groups (Fig. 8a).

Still within the one territory hypothesis, a simplistic view would be to consider that the Côa Valley group undertook special-purpose trips to gather those raw materials from sources inside its own territory (Fig. 7c). Besides the territory size problem, this hypothesis would ignore the existence of settlement in the areas where such sources are located, especially in the Portuguese Estremadura area, and the fact that the known data for special purpose trips in the Australian Western Desert indicate that they do not exceed $45 \mathrm{~km}$, travelled by motor vehicle (Gould and Saggers, 1985, Table 1), whereas the Côa Valley data implicates travel distances of 150 to $250 \mathrm{~km}$. However, the same Australian case-study identifies the existence of male-only trips of almost $300 \mathrm{~km}$ to neighbouring territories to gather raw material and visit sacred places. This case-study is an illustration of the fact that some territorially distinct hunter-gatherer groups often have direct access to resources outside their home ground (e.g. Renfrew, 1975) (Fig. 7d), because resources are not owned by individuals or groups and land 

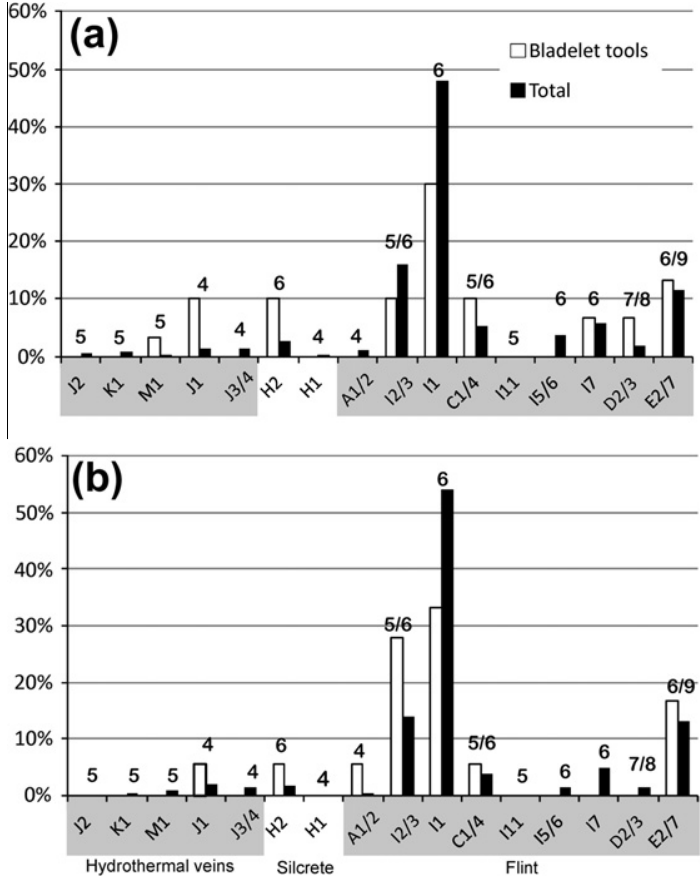

Fig. 5. Comparison between the proportion of all the fine-grained raw material found in Cardina's Recent Gravettian layers 4, 10 (a) and 4, 11 (b) (including subproducts of tool manufacture), and the proportion of its use in bladelet tools. (See Table 2 for raw material categories description and quality).

tenure is based on a common property regime (Lee and Daly, 1999). Even though land can be held by a kinship group, reciprocal access is the rule. This hypothesis obviates what seems an excessively vast territory, but the distances travelled within a single year continue to seem very long.

The longest documented straight-line distances covered during an annual range vary between 150 and $250 \mathrm{~km}$ (Labrador Innu, Baffinland, Labrador Inuit, pre-1885 Nunamiut), with one extreme case of $400 \mathrm{~km}$ (Ethen-eldèli Chipewyan) (Ellis, 2011). These examples all have in common its subarctic environment, the use of means of transportation, such as watercraft and dog sled (except for the Ethen-eldèli Chipewyan) and its purpose, caribou hunting.

Several reasons can justify frequent long-distance travels: low density population, extreme climatic conditions, high dependence of seasonal resources and colonisation processes. Archaeologically, some of the longest raw material displacements occurred during colonisation processes, such as early Clovis settlement (Ellis, 2011) and the Finnish Mesolithic (Hertell and Tallavaara, 2011). Such a context would only be acceptable for the earliest Upper Palaeolithic settlement in the Côa Valley and not for the 20,000 years sequence of stable raw material procurement structure observed, also revealing a perfect knowledge of spatially restricted local raw materials. As stated previously, a palaeoenvironental forcing is not supported by existing data.

The direct access hypothesis implies one approach the evidence as representing different groups or different territories. In this context we must also consider hypotheses involving exchange. Among egalitarian hunter-gatherers exchange has a completely different meaning than among societies with private property, since goods are shared in what has been defined as generalised reciprocity (Sahlins, 1972) or demand sharing (Peterson, 1993). With this in mind, a fifth scenario would be that, instead of having direct access to the sources, groups would reciprocally exchange raw materials. This exchange would either be made at the home bases of each other (Fig. 7e) or at territorial boundaries (Fig. 7f) (Renfrew, 1975). Several ethnographic examples of this kind of boundary
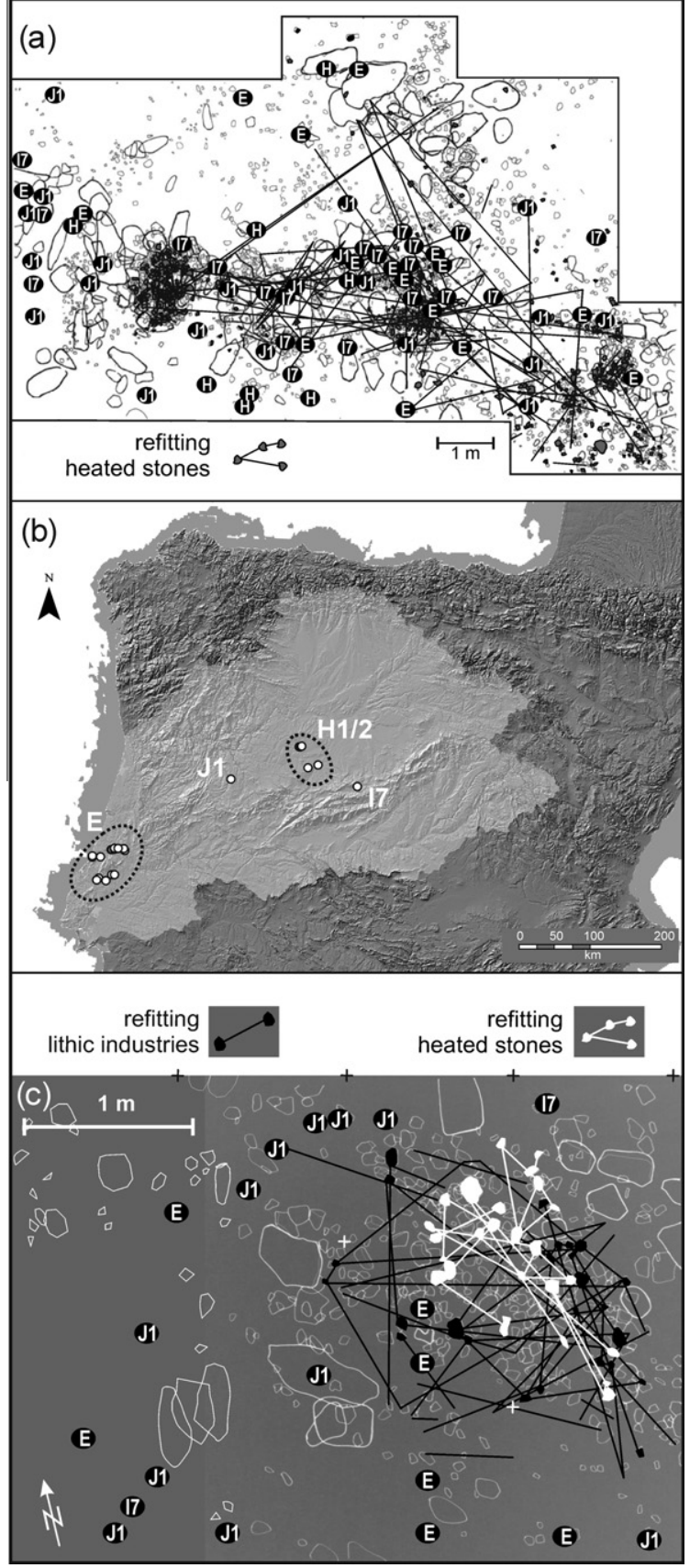

Fig. 6. Spatial distribution of refitted heated stones used in fire pits and of lithic raw material categories in the Gravettian layers of Olga Grande 4 (a) and Olga Grande 14 (c), with location of the fine-grained siliceous sources represented at both sites (b).

exchanges can be pointed out, notably amongst the Central Eskimo (Mauss and Beuchat, 1905), ! Kung (Lee, 1972) and North Andamanese (Kelly, 2005).

Finally, one can suppose that the studied area was homogeneously occupied by different bands practicing down-the-line exchange (Renfrew, 1975) (Fig. 7g). Thus, to arrive to the Côa Valley band, the different raw materials would have passed through different hands. This scenario presents some problems, mainly related to the fact that, despite systematic surveys in the Portuguese area, there are no known Upper Palaeolithic settlements in intermediate areas, even where raw material sources used in the Côa Valley are found (Fig. 1a). This result could be a product of a data bias due to the present state of research, as 


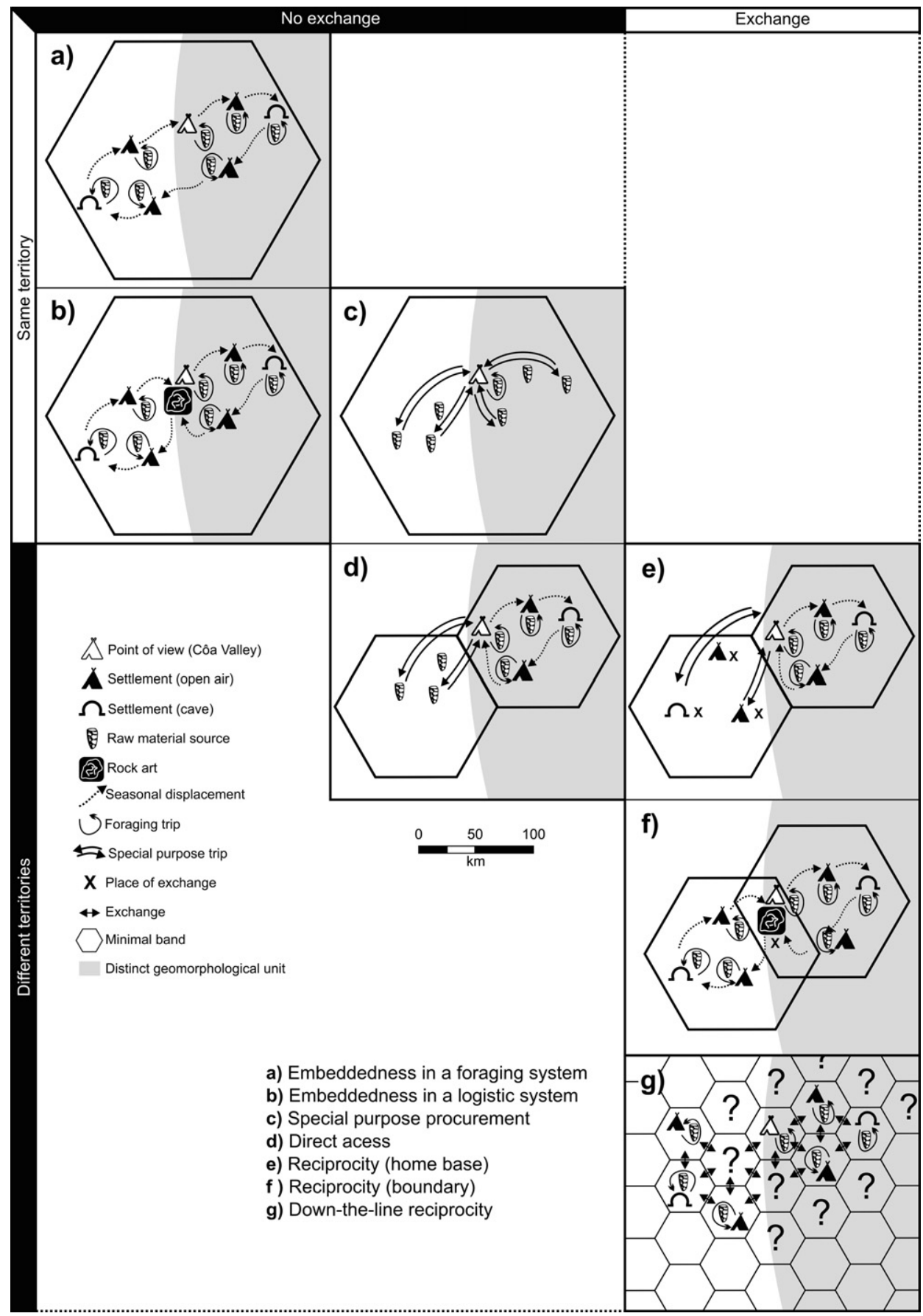

Fig. 7. Theoretical framework of cultural processes behind siliceous raw material long-distance displacements.

illustrated by the fact that prior to 1995 sites were completely unknown in the Côa Valley, but it could also equate with an Upper Palaeolithic reality. Moreover, if it was down-the-line exchange, the resulting raw material percentages would differ from the Côa Valley reality, predictably with closer sources better represented than distant ones.

The hypotheses presented are not necessarily mutually exclusive. One assumes that embeddedness and seasonal displacement are always part of the economic structure of hunter-gatherers. Special-purpose trips can exist in an embedded reality, and so can exchange.

The data suggest that direct procurement is most probable for the local and regional fine-grained siliceous rocks used in the Côa sites and for the high quality flint discarded systematically on the
Portuguese Estremadura sites. The spatial range of flint displacement in the sites located near flint sources of the Estremadura is similar to that defined by the hydrothermal micro-quartz vein varieties exploited in the Côa Valley (Fig. 8a, Table 3). The Côa Valley exotic raw material data point to a different scenario. Territory sizes, distances travelled, the archaeological record, and the presence of rock art in this natural geomorphological boundary suggest that the Côa Valley could have been an aggregation area, where dispersed bands would seasonally come together to fulfil their economic, social and cultural needs (Fig. 7f). The absence of sites between distant flint sources and the Côa Valley cannot be considered as definitive. A mixed hypothesis, such as the existence of intermediate bands that would acquire these raw materials via exchange, and bring it to the Côa Valley in the context of aggregation 

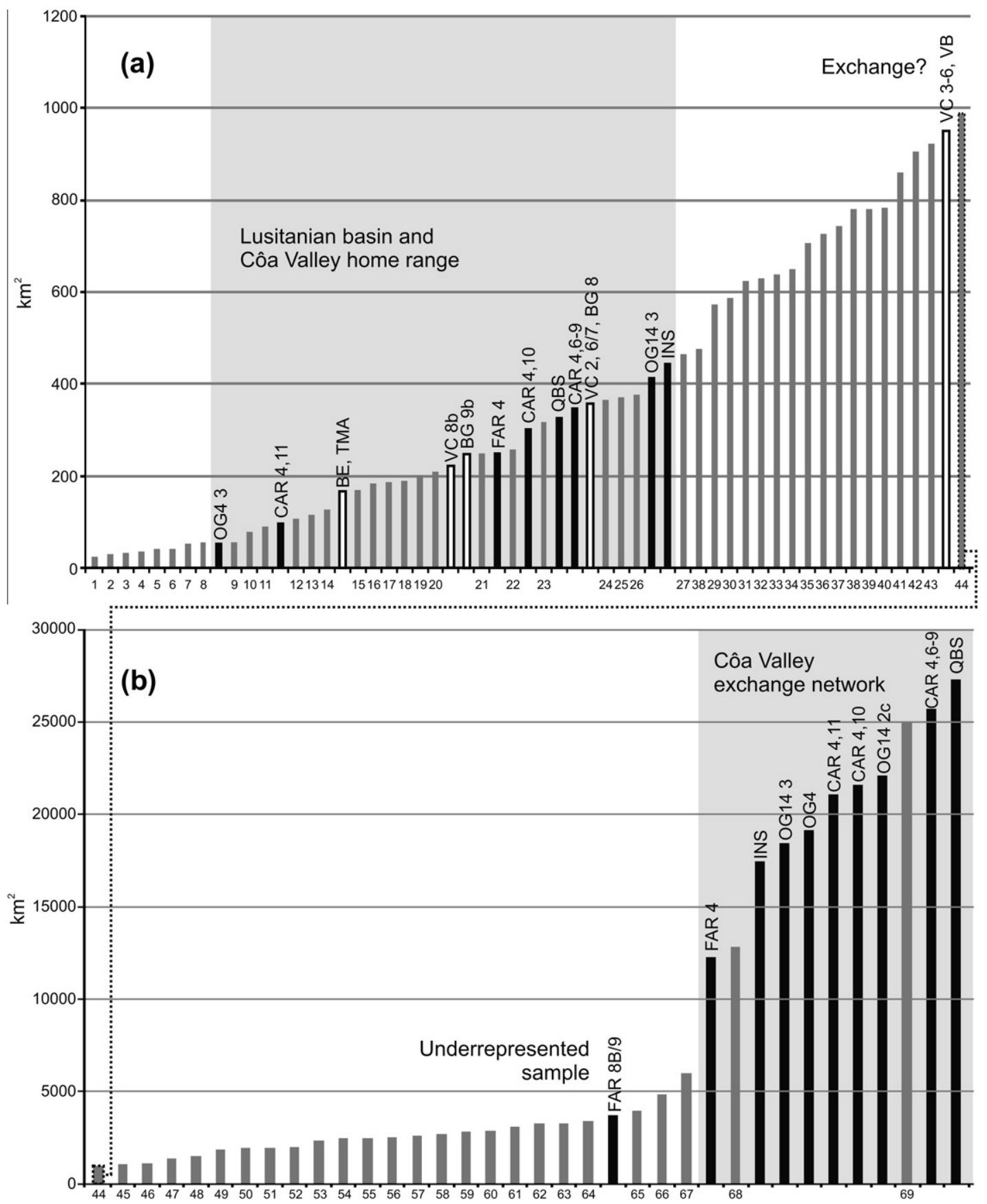

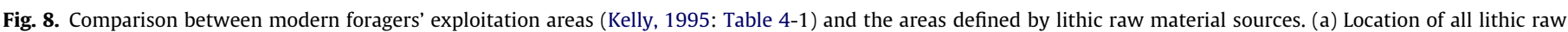

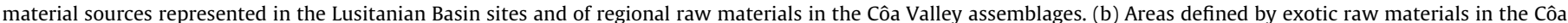

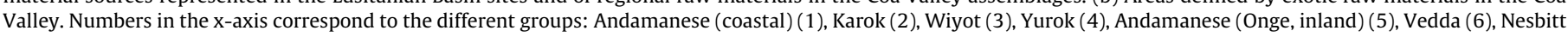

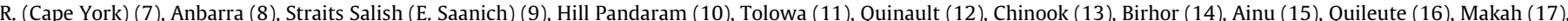

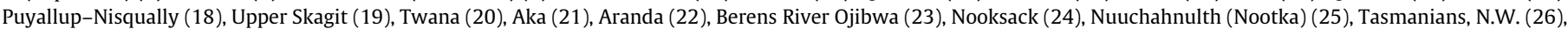

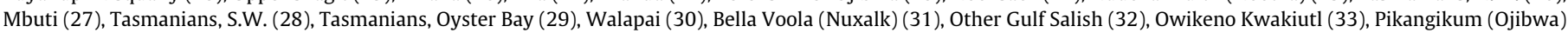

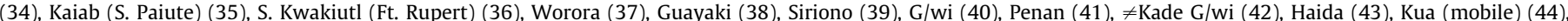

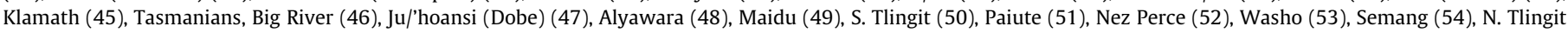

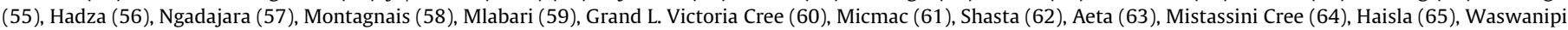
Cree (66), Netsilingmiut (67), Nunamiut (68), Baffinland Inuit (69). Values for groups 7, 27, 49, 61 and 68 correspond to mean areas.

or other, cannot be discarded. Like the Australian example previously exposed (Gould and Saggers, 1985), the exotic raw material could also have arrived in the context sporadic traveller's transit, for religious or marriage purposes. Marriage can be an important variable in this analysis. Dispersed and low density population require long-distance marriage networks needed for biological survival (Wobst, 1974), and so, mobility can be regarded as a reproductive strategy (Macdonald, 1998). Beyond a certain threshold, the percentage of marriages decreases with distance, since it ceases to be advantageous (Hertell and Tallavaara, 2011). This marriage or kinship network has been defined as a maximum band (meta-group), which integrates several minimum bands (residential groups)(Wobst, 1974). The regulated exchange of mates and resources among hunter-gatherers, as well as co-residence patterns 

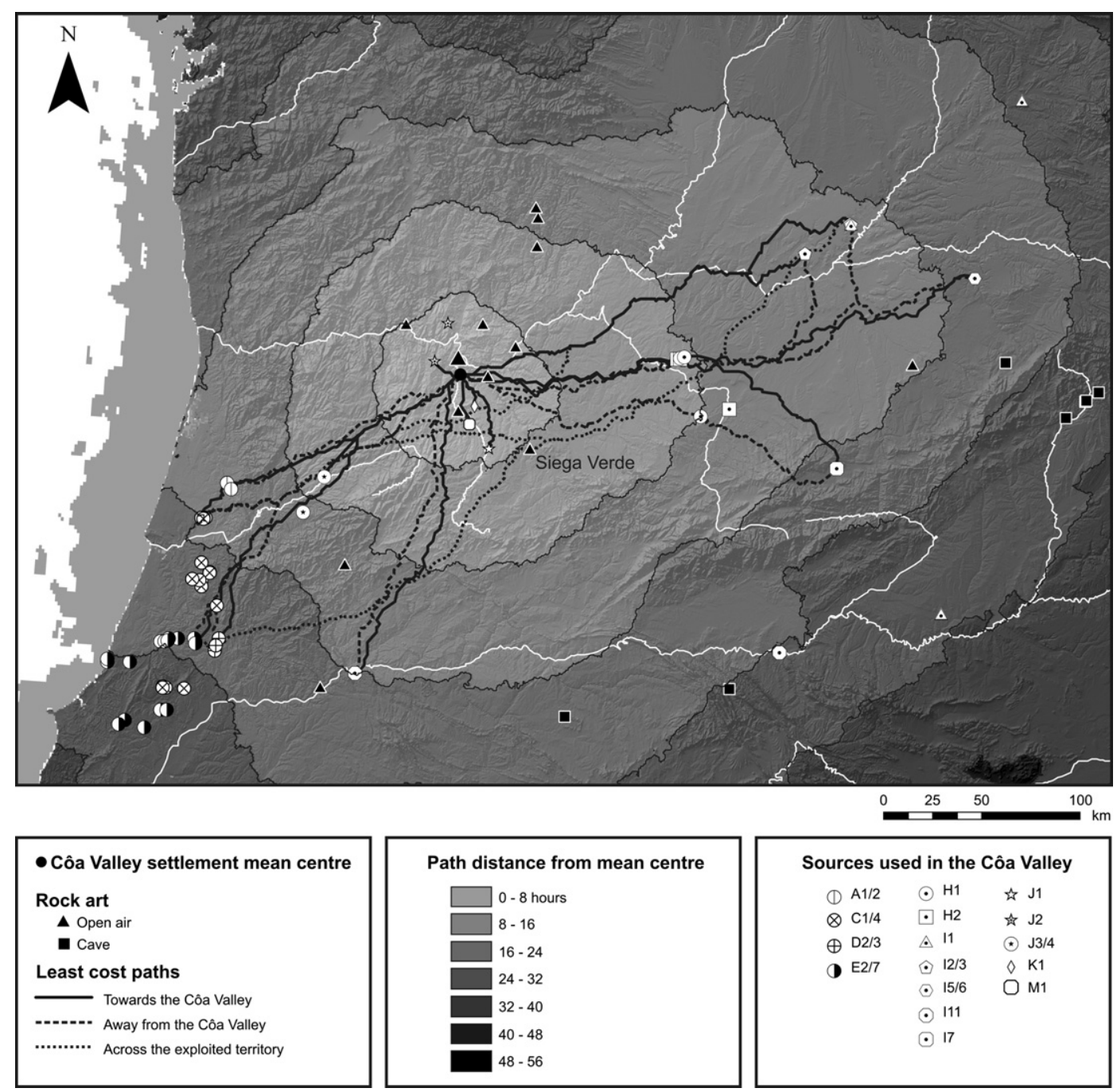

Fig. 9. Least-cost paths between raw material sources and settlement destinations.

have been recently identified as keys for the genetic variability of residential communities and the complexity of human social structure through the promotion of coalitions and alliances (Hill et al., 2011; Walker et al., 2011). The existence of marriages among an endogamous maximal band and other rites of passage may occur in aggregation contexts. However, this cannot be understood on a sporadic basis. The fact that exotic raw materials are constantly present in non negligible quantities and respond to permanent needs is also an argument against casual traveller's transit.

\section{An aggregation model for the Côa Valley}

Applying a central place model to our study (Wobst, 1974; Whallon, 2006), the smallest spatial level of the local band would correspond to the local sources ranges documented by lithic raw material displacement in the Portuguese Estremadura and the Côa Valley. The permanent settlement of the Côa area is justified by the knowledge evident in the use of all local raw material types, even those with very restricted sources (J1, J2, M1 and K1).

Ethnographic data and heuristic modelling postulate that such minimum bands in low-population-density areas are not self-sufficient and cannot survive in the long-term (Whallon, 2006). Demography, but also variation in the availability of resources, requires the existence of "safety nets" composed of maximum or regional bands grouping several adjacent minimum or local bands
(Whallon, 2006). Based on the raw materials found in the Côa assemblages we propose that, in this case, the Côa Valley maximum band included Portuguese Estremadura and Northern Meseta local groups and that these areas were part of the same social network of exchange. In such an interpretation, we suggest that the displacement of flint from the Meseta and Portuguese Estremadura was realised by two or more different bands, and toolstone was exchanged and used in the Côa region.

The orographic characteristics of the Côa region suggest its classification as an ecotone area (Farina, 2006): from East to West, it corresponds to the end of the Meseta plateau ( $600 \mathrm{~m})$, with several quartzitic residual reliefs ( $\sim 900 \mathrm{~m})$ and cut by the deeply incised Douro and Côa valley river bottoms ( $\sim 115 \mathrm{~m})$, bounded to the West by the rugged Western Mountains, with a $200 \mathrm{~m}$ elevation difference.

Among mobile foragers, other than blocks of land, territories can be defined by paths, as exemplified by Mbuti pygmies, whose territories are determined by narrow paths which connect several hunting camps (Ichikawa, 1978). Least-cost paths from and to exotic raw material sources allowed us to better understand land-use and mobility in the study area. With one exception, paths from and to Eastern flint sources run through the exact area where the outcrops yielding silcrete sources ( $\mathrm{H} 1$ and $\mathrm{H} 2$ ) are located (Fig. 9). The exact same scenario is verified with South-western flint sources, where the paths defined pass in the vicinities of hydrothermal 
fine-grained quartz veins (J4). These sources were used in the Côa Valley throughout the entire Upper Palaeolithic sequence, and are located at $\sim 16 \mathrm{~h}$ distance, and in the same direction as the flint sources located at $\sim 32 \mathrm{~h}$. These results derived from GIS analysis locate these lesser quality sources $(\mathrm{H} 1, \mathrm{H} 2$ and $\mathrm{J} 4)$ used in the Côa Valley approximately halfway along the least-cost path between this area and the flint sources. This fact is consistent with a scenario in which flint arrived in the Côa Valley in the context of long-distance displacement by distinct human groups coming from two, large, outside areas. Nevertheless, one cannot completely discard a second hypothesis, where groups originating from this intermediate area could have brought flint from the Northern Meseta and the Portuguese Estremadura, after obtaining it through exchange. This cost time analysis also reveals that, even though the distance between the Estremadura sources and the Côa Valley is smaller as the crow flies, topographic constraints to movement facilitate displacement towards the Northern Meseta, therefore making it closer.

To test the hypothesis that the Côa Valley could have been a natural crossing point between the Northern Meseta and Portuguese Estremadura, least-cost paths between two extreme sources from the Meseta, and two others from Estremadura, were produced. Even though these origin and destiny points are arbitrary, since there is no evidence of Northern Meseta raw material in the Estremadura Upper Palaeolithic sites, and vice versa, the least-cost paths show that, when moving between these two large regions, the Côa Valley is a natural passageway. All of the defined paths run less than $50 \mathrm{~km}$ south of the rock art area. Only one is farther out, even though it runs through the neighbouring Upper Palaeolithic rock art site of Siega Verde.

The Côa Valley region is located between two large geographical entities, the Northern Meseta and the Western Mountains (Ferreira, 1978). Historically, the Côa River defined the border between the kingdoms of Portugal and Castile-Leon until 1297, when a treaty incorporated the Ribacoa area, its right bank, in the Portuguese territory. This area, which integrated Meseta land in the Portuguese borders, was an historical pathway between the Atlantic coast of Iberia and the rest of Europe via the Iberian hinterland. In 1282, Elisabeth of Aragon crossed it to marry Denis of Portugal in Trancoso. In 1551, an elephant, offered as a wedding present by John III of Portugal to Archduke Maximilian, passed through Castelo Rodrigo on its way to Austria. In 1810, during the third Napoleonic invasion, the French forces entered Portugal through here, with fighting taking place in the Côa river banks.

Based on the raw material sourcing data and the geographical specificity of this area, we propose that the Côa Valley corresponded to a natural geographical transition zone that can be recognised from the Upper Palaeolithic settlement. This area would be an inclusive one that corresponded to the phase of aggregation or "public life" (Mauss and Beuchat, 1905; Lee, 1972) in a land-use pattern of fusion-fission (Fig. 7f). These moments of aggregation can be promoted by ecological factors (Steward, 1936), such as seasonal resource concentration, but present major opportunities social and ritual activities (Lee, 1972). These moments are particularly important for the exchange of long-distance products, marriage brokering and adoption, but also for rites of passage and other collective rituals based on singing and dancing (Lee, 1972; Kelly, 2005). These are moments of intensified social life, that will end inevitably due to the increasing difficulty in obtaining resources for a large number of people, and the increasing potential conflict (Lee, 1972), even though they can also be seen as a means to prevent intergroupal violence, in the case of territorial disputes (Kelly, 2005).

The fact that the Côa Valley presents the largest concentration of open-air Palaeolithic rock art known today is of crucial importance in our interpretation. Firstly, it is located along the Meseta's western limit, as is a significant proportion of Iberian open-air rock art (Fig. 1a). Secondly, several authors have highlighted the social nature of rock art, as a record of exchanges between bands, namely via the exchange of partners or the formation of coalitions (Laming-Emperaire, 1972), the evidence of rites of passage (Owens and Hayden, 1997), and the materialisation of aggregation sites (Conkey, 1980). The outside nature of open-air rock art sites, such as in the Côa Valley, makes this interpretation even more evident, as has already been proposed (Baptista and García Díez, 2002; Baptista et al., 2009; Santos, in press). The identification of this complex of rock art sites has shed new light on the Pleistocene artistic phenomenon, which nowadays can be seen an eminently open-air phenomenon, even though, until recently, it was perceived as cave art because of differential preservation (Bahn, 1995).

The lithic raw material sourcing data add to the interpretation of this area's rock art. If raw materials circulate primarily among maximum bands, other symbolic items, such as shells, may prove even wider contacts between adjacent maximum bands (Whallon, 2006). The nature of the Côa Valley archaeological record is not amenable to the assessment of this type of contacts, but its rock art is different. The different phases of the Côa Valley rock art attest stylistic commonalities with distant regions implying contacts at an even broader scale than that suggested by the lithic raw material evidence (Zilhão, 2003). The pre-Magdalenian phase, dated archaeologically at Fariseu (Aubry and Sampaio, 2008a,b; Aubry, 2009), is stylistically close to examples from North-eastern France to Southern Spain (Guy, 2002; Santos, in press). Also dated archaeologically, a Final Magdalenian/Azilian phase, present not only in rock panels but also on portable art recovered in a dated context (11,000-12,000 BP) at the Fariseu site (Aubry, 2009; Aubry et al., 2010), shows stylistic affinities with Peña de Estebanvela in the Northern Meseta as well as with sites in South-eastern Spain and Southern France (García Díez and Aubry, 2002; Baptista and Santos, 2010). An intermediate, still archaeologically undated, Middle/Upper Magdalenian phase also presents long-distance stylistic relations with the Northern Meseta (Domingo García) and the North of Spain (Santos, in press).

Stylistic affinities are not coincidences and indicate contacts outside the immediate safety nets. They are beyond the threshold of economic reason and attest the symbolic dimension of human relations. As social networks enlarge, emotional and material exchange and reciprocity diminishes, but symbol and style gain importance for communication and identification purposes (Wobst, 1977).

The Côa Valley lithic tools system integrating systematically exotic raw materials reveals a broad social network of Upper Palaeolithic hunter-gatherer societies. These societies produced and used rock art, which due to its symbolic nature puts in evidence a second and largest level of social networking.

\section{Conclusion}

Data obtained from the study of the Côa Valley lithic raw material procurement throughout the Upper Palaeolithic, its technological use, and comparisons with contemporary occupation in cave, rock-shelter and open-air sites in central Portugal allow us to assess hunter-gatherer mobility and to reconstruct a large scale three level social network.

The first level corresponds to the home ranges of minimum bands, testified by the embedded supply of local and regional lithic raw materials, identified both on the Côa Valley and the Portuguese Estremadura sites.

The second level is attested by the presence of exotic raw material in the Côa Valley. The geological characterisation and identification of the sources represented in the Côa Valley assemblages, 
and the differential use of the lithic raw materials, indicate that this region was the destiny of exotic materials arriving from two geographically opposed regions, the Northern Meseta and Portuguese Estremadura. Regional geomorphology and GIS least-cost paths place the Côa Valley in a transition zone between the Iberian Plateau and the rugged Western Mountains that reach the Atlantic (Fig. 9), and show that it is a natural pathway between the Atlantic coast and the Iberian hinterland, as otherwise historically documented. The presence in the area of the largest concentration of Upper Palaeolithic open-air rock art currently known led us towards a sociocultural model, which interprets the exotic raw materials as the product of use and exchange between members of one endogamous maximum band (Wobst, 1974) in the context of aggregation events. We perceive the raw material data and the rock art as the materialisation of complex social and cultural behaviours that should have also included the exchange of other goods and mates, but also group activities. Finally, the presence of rock art in the area, contemporary with the long duration Upper Palaeolithic settlement, and its stylistic links with sites from an even wider area, puts in evidence the existence of a third level, beyond the maximum group, related to symbolic items (Whallon, 2006).

From the long duration of the rock art tradition and the constant representation of the same basic lithic raw material sources we could infer a stable system, lasting from before the LGM to the Lateglacial. However, fine-grained analysis argues for dynamic changes within this general structure, as observed through an increase in the ratios of sources below the median of the exotic raw material distances $(197 \mathrm{~km})$ and procured from the Southwest. The network seems to be constrained. This fact could be interpreted as a result of population growth, which is also suggested by an increase in the rabbit to ungulate ratio, as previously mentioned. Moreover, the Côa Valley social network shifts focus, with Eastern inland losing its relative significance in favour of South-western coastal areas. This fact could account for the increased exploitation of aquatic resources during the Magdalenian in Estremadura (Bicho et al., 2003). Territorial constriction, population increase and climate change all point to subsistence stress and a path towards sedentism and land tenure (Kelly, 1992).

Changes in the Côa Valley rock art are concurrent with the changes identified in lithic raw material. Lateglacial art is much more private and discrete than the pre-Magdalenian phase, in terms of size, techniques, placement and overall visibility (Aubry and Luís, 2012; Santos, in press).

Except for Portugal, where site counts are heavily dominated by open-air localities in all periods (Zilhão, 1997), Upper Palaeolithic research in Iberia has basically followed the cave and flint paradigm, focusing mainly on carbonate rocks formed in a marine environment (Northern, Western, South and South-eastern coasts). In this respect, the Côa Valley opens new avenues for the study of Upper Palaeolithic land use in the Iberian hinterland, far away from flint sources. This natural constraint and the cultural choices in stone tool production enable us to study long-distance relations with areas where this fundamental raw material was naturally available, and to integrate it in a cultural system where rock art was produced and used. However, a geoarchaeological approach has shown that site formation and preservation throughout the Upper Palaeolithic is not homogenous and highly dependent on environment changes and sedimentary processes (Zilhão, 2003; Aubry et al., 2010, 2012). The Côa Valley Upper Palaeolithic settlement still remains an isolated island in the sea of the Iberian inland. Additional research, namely in the Northern Meseta and in Central Portugal, where no settlement is known, despite the existence of raw material sources used in the Côa Valley, is needed to obtain a sharper image of the variability in the exploration of abiotic resources and in social networking patterns over the 20,000 years' duration of the regional Upper Palaeolithic.

\section{Acknowledgments}

This study was funded by the Instituto Português de Arqueologia, the Instituto de Gestão do Património Arquitectónico e Arqueológico and the Departament de Prehistòria, Història Antiga i Arqueologia, SERP, Universitat de Barcelona. The survey and study of samples from the Serra dos Candeeiros region and the survey of Miocene flint of Tordesillias-Valladolid area was funded by the PTDC/HIS-ARQ/098164/2008 - Middle Palaeolithic Archaeology of the Almonda Karstic System F.C.T: project coordinated by João Zilhão. We would like to thank João Zilhão for constructive comments on an early version of the manuscript, the two anonymous reviewers for careful reading and valuable insights, and John Willman for abstract wording revision.

\section{Appendix A. Supplementary data}

Supplementary data associated with this article can be found, in the online version, at http://dx.doi.org/10.1016/j.jaa.2012.05.003. These data include Google maps of the most important areas described in this article.

\section{References}

Almeida, F., 2000. The Terminal Gravettien of Portuguese Estremadura. PhD Dissertation. Southern Methodist University.

Almeida, F., Araújo, A.C., Aubry, T., 2003. Paleotecnologia lítica: Dos objectos aos comportamentos. In: Mateus, J. E., Moreno-García, M. (Eds.), Paleoecologia Humana e Arqueociências: Um programa multidisciplinar para a arqueologia sob a tutela da cultura. Instituto Português de Arqueologia (Trabalhos de Arqueologia 29), Lisboa, pp. 299-349.

Almeida, F., Angelucci, D., Gameiro, C., Correia, J., Pereira, T., 2004. Novos dados para o Paleolítico Superior final da Estremadura Portuguesa: Resultados preliminares dos trabalhos arqueológicos de 1997-2003 na Lapa dos Coelhos (Casais Martanes, Torres Novas). Promontoria 2 (2), 157-192.

Almeida, F., Matias, H., Carvalho, R., Pereira, T, Pinto, A., Holliday, T., 2010. New data on the transition from the Gravettian to the Solutrean on Portuguese Estremadura. In: Poster presented to the Paleoanthropology Society Meetings, Saint Louis (April).

Amante, C., Eakins, B.W., 2009. ETOPO1 1 Arc-Minute Global Relief Model: Procedures, Data Sources and Analysis. NOAA Technical Memorandum NESDIS NGDC-24.

Angelucci, D., Zilhão, J., 2009. Stratigraphy and formation processes of the Late Pleistocene deposit at Gruta da Oliveira, Almonda karstic system, Torres Novas, Portugal. Geoarchaeology 24 (3), 277-310.

Armenteros, A.I., 2000. Estratigrafia y Sedimentologia del Neogeno del Sector Suroriental de la Depression del Duero. Diputación de Salamanca (Serie Castilla y León 1), Salamanca.

Aubry, T., 2001. L'occupation de la basse vallée du Côa pendant le Paléolithique supérieur. In: Zilhão, J., Aubry, T., Carvalho, A.F. (Eds.), Les premiers hommes modernes de la péninsule ibérique (Actes du Colloque de la Commission VIII de l'UISPP. Vila Nova de Foz Côa, 22-24 Octobre 1998). Instituto Português de Arqueologia (Trabalhos de Arqueologia 17), Lisboa, pp. 253-273.

Aubry, T., 2002. Le contexte archéologique de l'art paléolithique à l'air libre de la vallée du Côa. In: Sacchi, D. (Ed.), L'Art Paléolithique à l'Air Libre: Le Paysage Modifié par l'Iimage (Tautavel, Campôme, 7-9 octobre 1999). GAEP; GÉOPRE, Saint-Estève, pp. 25-38.

Aubry, T., 2009. 200 séculos de história do Vale do Côa: Incursões na vida quotidiana dos caçadores-artistas do Paleolítico. IGESPAR, I.P. (Trabalhos de Arqueologia 52), Lisboa.

Aubry, T., Araújo Igreja, M., 2009. Economy of lithic raw material during the Upper Paleolithic of the Côa Valley and the Sicó Massif (Portugal): technological and functional perspectives. In: Araújo Igreja, M., Clemente Conte, I. (Eds.) Proceedings of the Workshop Functional Studies of Non Flint Stone Tools: Methodological Improvements and Archaeological Inferences. Lisbon, 23-25 May 2008. <http://www.arte-coa.pt/Ficheiros/Bibliografia/1203/1203.pt.pdf> (accessed 10.01.12)

Aubry, T., Luís, L., 2012. Umwelt und Gesellschaft der paläolithischen Freilandkunst im Côa-Tal (Portugal). In: Dally, O., Moraw, S., Ziemssen, H. (Eds.), Bild-RaumHandlung: Perspektiven der Archäologie.De Gruyter (Topoi 11), Berlin, pp. 5183.

Aubry, T., Mangado Llach, J., 2003a. Modalidades de aprovisionamento em matériasprimas líticas nos sítios do Paleolítico superior do Vale do Côa: Dos dados à interpretação. In: Mateus, J.E., Moreno-García, M. (Eds.), Paleoecologia Humana e Arqueociências: Um programa multidisciplinar para a arqueologia sob a tutela da cultura. Instituto Português de Arqueologia (Trabalhos de Arqueologia 29) Lisboa, pp. 340-342. 
Aubry, T., Mangado Llach, X., 2003b. Interprétation de l'approvisionnement en matières premières siliceuses sur les sites du Paléolithique supérieur de la vallée du Côa (Portugal). In: Les Matières Premières Lithiques en Préhistoire (Table ronde internationale organisée à Aurillac, Cantal, du 20 au 22 juin 2002). Association Préhistoire du Sud-ouest (Préhistoire du Sud-ouest, Supplément no. 5), Carcassone, pp. 27-40.

Aubry, T., Mangado Llach, J., 2006. The Côa Valley (Portugal): Lithic raw material and the reconstruction of Upper Palaeolithic settlement patterns. In: Bressy, C., Burke, A., Chalard, P., Martin, H. (Eds.), Notions de Territoire et de Mobilité: Exemples d'Europe et des Premières Nations en Amérique du Nord avant le contact européen. Université de Liège (ERAUL 116), Liège, pp. 41-49.

Aubry, T., Sampaio, J., 2008a. Chronologie et contexte archéologique des gravures paléolithiques de plein air de la Vallée du Côa (Portugal). In: de Balbín Behrmann, R. (Ed.), Arte Prehistórico al aire libre en el Sur de Europa. Junta de Castilla y Léon, pp. 211-223.

Aubry, T., Sampaio, J.D., 2008b. Fariseu: New Chronological Evidence for Open-Air Palaeolithic Art in the Côa Valley (Portugal). Antiquity 82 (316) Project Gallery. <http://antiquity.ac.uk/ProjGall/aubry316> (accessed 10.01.12).

Aubry, T., Moura, M.H., Zilhão, J., 1994. Dados preliminares sobre a organização estratigráfica da Buraca Grande do Vale do Poio Novo (Redinha). Memórias Notícias, Publicações do Museu de Mineralogia e Geologia da Universidade de Coimbra 114, 119-132.

Aubry, T., Fontugne, M., Moura, M.H., 1997. Les occupations de la grotte de Buraca Grande depuis le Paléolithique supérieur et les apports de la séquence holocène à l'étude de la transition Mésolithique/Néolithique au Portugal. Bulletin de la Société Préhistorique Française 94 (2), 182-190.

Aubry, T., Brugal, J.-P., Chauvière, F.X., Figueiral, I., Moura, M.H., Plisson, H., 2001 Modalités d'occupation au Paléolithique supérieur dans la grotte de Buraca Escura (Redinha, Pombal, Portugal). Revista Portuguesa de Arqueologia 4 (2), 19-46.

Aubry, T., Mangado Llach, X., Sellami, F., Sampaio, J.D., 2002. Open-air rock-art, territories and modes of exploitation during the Upper Paleolithic in the Côa Valley (Portugal). Antiquity 76 (291), 62-76.

Aubry, T., Chauvière, F.-X., Mangado Llach, X., Sampaio, J.D., 2003. Constitution, territoires d'approvisionnement et fonction des sites du Paléolithique supérieu de la basse vallée du Côa (Portugal). In: Vasil'ev, S.A., Soffer, O., Koslowski, J. (Eds.), Perceived Landscapes and Built Environments: The Cultural Geography of Late Paleolithic Eurasia. Archaeopress (BAR International Series 1122), Oxford, pp. 83-92.

Aubry, T., Mangado Llach, X., Fullola, J. M., Rosell, L., Sampaio, J. D., 2004. The Raw Material Procurement in the Upper Palaeolithic Settlements of the Côa Valley (Portugal): new data concerning modes of resource exploitation in Iberia. In Smyntyna, O.V. (Ed.), The Use of Living Space in Prehistory: Papers from a Session held at the European Association of Archaeologists Sixth Annual Meeting in Lisbon 2000. Archaeopress (BAR International Series 1224), Oxford, pp. 37-50.

Aubry, T., Luís, L., Sampaio, J.D., 2007. Primeira datação absoluta para a arte paleolítica ao ar livre: Os dados do Fariseu (Vila Nova de Foz Côa). Al-Madan 14 $48-52$.

Aubry, T., Almeida, M., Dimuccio, L., Cunha, L., Neves, M.J., 2008. Archives géoarchéologiques des grottes et abris sous roches du Massif de Sicó (Portugal). In: Brandão, J., Calado, C., Sá Couto, F. (Eds.), Actas do Simpósio Ibero-Americano: Património Geológico, Arqueológico e Mineiro em Regiões Cársicas". SEDPGYM, Batalha, pp. 239-251.

Aubry, T., Dimuccio, L.A., Bergadà, M.M., Sampaio, J.D., Sellami, F., 2010. Palaeolithic engravings and sedimentary environments in the Côa River Valley (Portugal): implications for the detection, interpretation and dating of open-air rock art Journal of Archaeological Science 37 (12), 3306-3319.

Aubry, T., Luís, L., Dimuccio, L.A., 2012. Nature vs. culture: present-day spatial distribution and preservation of open-air rock art in the Côa and Douro River Valleys (Portugal). Journal of Archaeological Science 39 (4), 848-866.

Bahn, P.G., 1995. Cave art without the caves. Antiquity 69, 231-237.

Bailey, G., 2007. Time perspectives: palimpsest and the archaeology of time. Journa of Anthropological Archaeology 26, 198-223.

Baptista, A.M., García Díez, M., 2002. L'art paléolithique dans la vallée du Côa (Portugal): La symbolique dans l'organisation d'un sanctuaire en plein air. In: Sacchi, D. (Ed.), L'art paléolithique à l'air libre: Le paysage modifié par l'image (Tautavel, Campôme, 7-9 octobre 1999). GAEP, GÉOPRE, Saint-Estève, pp. 187 205.

Baptista, A.M., Santos, A.T., 2010. Confronting two sceneries on the same stage: From Gravettian-Solutrean to Magdalenian in Penascosa/Quinta da Barca (Vila Nova de Foz Côa, Portugal). In: Bettencourt, A.M.S., Sanches, M.J., Alves, L.B Fábregas Valcarce, R. (Eds.), Conceptualising Space and Place. On the Role of Agency, Memory and Identity in the Construction of Space from the Upper Palaeolithic to the Iron Age in Europe Archaeopress (BAR International Series 2058), Oxford, pp. 61-69.

Baptista, A.M., Santos, A.T., Correia, D., 2009. O santuário arcaico do Vale do Côa: Novas pistas para a compreensão da estruturação do bestiário gravettense e/ou gravetto-solutrense. In: Balbín Behrmann, R. (Ed.), Actas do Curso de Arte Rupestre al Aire Libre: Investigacíón, Protección, Conservación y Difusón. Junta de Castilla y León (Documentos PAHIS), Valladolid, pp. 89-144.

Bard, E., Rostek, F., Turon, J.L., Gendreau, S., 2000. Hydrological impact of Heinrich events in the subtropical Northeast Atlantic. Science 289, 1321-1324.

Bicho, N.F., 2000. Paleolithic occupation and environment of central and southern Portugal during Isotopic Stages 2 and 3. In: Vermeersch, P., Renault-Miskovsky, J. (Eds.), European Late Pleistocene Isotopic Stages 2 \& 3: Humans, their Ecology and Cultural Adaptations. Université de Liège, Liège, pp. 43-56.
Bicho, N.F., Belcher, W., Haws, J.A., Markova, A., Hockett, B.S., 2003. Paleoecologia e ocupação humana da Lapa do Picareiro: resultados preliminares. Revista Portuguesa de Arqueologia 6 (2), 49-82.

Binford, L.R., 1979. Organization and formation processes: looking at curated technologies. Journal of Anthropological Research 35 (3), 255-273.

Binford, L.R., 1980. Willow smoke and dogs' tails: hunter-gatherer settlement systems and archaeological site formation. American Antiquity 45 (1), 4-20.

Binford, L.R., 1983. In Pursuit of the Past: Decoding the Archaeological Record. Thames \& Hudson, London.

Blanco, J.A., Armenteros, I., Huerta, P., 2008. Silcrete and alunite genesis in alluvial palaeosols (late Cretaceous to early Palaeocene, Duero basin, Spain). Sedimentary Geology 211, 1-11.

Bond, G.C., Broecker, W., Johnsen, S., McManus, J., Labeyrie, L., Jouzel, J., Bonani, G., 1993. Correlations between climate records from North Atlantic sediments and Greenland ice. Nature 365, 143-147.

Bond, G., Showers, W., Cheseby, M., Lotti, R., Hajdas, I., Bonani, G., 1997. A pervasive millennial-scale cycle in North Atlantic Holocene and glacial climates. Science 278, 1257-1266.

Bustillo, M.A., 1976. Estudio Petrológico de las rocas silíceas miocenas de la Cuenca del Tajo. Estudios Geológicos 32, 451-497.

Bustillo, M.A., Pérez-Jiménez, J.L., 2005. Características diferenciales y génesis de los niveles silíceos explotados en el yacimiento arqueológico de Casa Montero (Vicálvaro, Madrid). Geogaceta 38, 243-246.

Cacho Quesada, C., Yravedra Sainz de los Terreros, J., Jordá Pardo, J.F., Ripoll López, S., Muñoz Ibáñez, F.J., 2007. La Peña de Estebanvela: Reconstrucción paleoambiental, recursos alimenticios, cronología y contexto del registro arqueológico: Contactos con la Cornisa Cantábrica y el ámbito pirenaico. In: Cacho Quesada, C., Ripoll López, S., Muñoz Ibáñez, F.J. (Eds.), La Peña de Estebanvela (Estebanvela-Ayllón, Segovia): Grupos Magdalenienses en el Sur del Duero. Junta de Castilla y León Consejería de Cultura y Turismo, Valladolid, pp. 427-444.

Carvalho, G.S., 1946. Sílex dos depósitos da Orla Mesozóica Ocidental: Elementos para o estudo da sua petrografia e da sua génese. Memórias e Notícias, Publicações do Museu de Mineralogia e Geologia da Universidade de Coimbra 18, 1-39.

Cayre, O., Lancelot, Y., Vincent, E., Hall, M.A., 1999. Paleoceanographic reconstruction from the plantonic foraminifera off the Iberian margin: temperature, salinity and Heinrich events. Palaeoceanography 14, 384-396.

Cerveira, A., 1951. Sobre a metalogenia do urânio em Portugal. Boletim da Sociedade Geológica de Portugal 8 (3), 141-182.

Combourieu Nebout, N., Turon, J.-L, Zahn, R, Capotondi, L, Londeix, L, Pahnke, K. 2002. Enhanced aridity and atmospheric high-pressure stability over the western Mediterranean during the North Atlantic cold events of the past 50 k.y. Geology 30, 863-866.

Conkey, M., 1980. The identification of prehistoric hunter-gatherer aggregation sites: the case of. Altamira. Current Anthropology 21 (5), 609-630.

Cunha, L., 1990. As Serras Calcárias de Condeixa-Sicó-Alvaiázere: Estudo de Geomorfologia. INIC (Geografia Física 1), Coimbra.

Davidson, I., 1986. The geographical study of Late Palaeolithic stages in Eastern Spain. In: Bailey, G., Callow, P. (Eds.), Stone Age Prehistory: Studies in Memory of Charles MacBurney. Cambridge University Press, Cambridge, pp. 95-118.

Davis, S.J.M., 2002. The mammals and birds from the Gruta do Caldeirão, Portugal. Revista Portuguesa de Arqueologia 5 (2), 29-98.

de Abreu, L., Shackleton, N.J., Scoenfeld, J., Hall, M., Chapman, M., 2003. Millennialscale oceanic climate variability off the Western Iberian margin during the last two glacial periods. Marine Geology 196, 1-20.

de Vicente, G., Cloetingh, S., VanWees, J.D., Cunha, P.P., 2011. Tectonic classification of Cenozoic Iberian foreland basins. Tectonophysics 502 (1-2), 38-61.

Debret, M., Bout-Roumazeilles, V., Grousset, F., Desmet, M., McManus, J.F., Massei, N., Sebag, D., Petit, J.-R., Copard, Y., Trentesaux, A., 2007. The origin of the 1500year climate cycles in Holocene North-Atlantic records. Climates of the Past 3 , 569-575.

Demars, P.-Y., 1982. L'utilisation du silex au Paléolithique supérieur : Choix, approvisionnement, circulation : L'exemple du Bassin de Brive. CNRS (Cahiers du Quaternaire 5), Paris.

d'Errico, F., Sánchez Goñi, M.F., 2003. Neanderthal extinction and the millennial scale climatic variability of OIS 3. Quaternary Science Review 22, 769-788.

Ellis, C., 2011. Measuring Paleoindian range mobility and land-use in the Great Lakes/Northeast. Journal of Anthropological Archaeology 30, 385-401.

Fábrega Álvarez, P., Parcero Oubiña, C., 2007. Proposals for an archaeological analysis of pathways and movement. Archeologia e Calcolatori 18, 121-140.

Farina, A., 2006. Principles and Methods in Landscape Ecology: Toward a Science of Landscape. Springer, Dordrecht.

Farr, T.G. et al., 2007. The Shuttle Radar Topography Mission. Reviews of Geophysics 45, RG2004. http://dx.doi.org/10.1029/2005RG000183.

Féblot-Augustins, J., 1997. La circulation des matières-premières au Paléolithique. Université de Liège (ERAUL 75), Liège.

Féblot-Augustins, J., 1999. La mobilité des groupes paléolithiques. Bulletin et Mémoires de la Société d'Anthropologie de Paris 11 (3-4), 219-260.

Féblot-Augustins, J., Perlès, C., 1992. Perspectives ethnoarchéologiques sur l'échange à longue distance. In: Ethnoarchéologie: Justification, Problèmes, Limites (XIIe Rencontres Internationales d'Archéologie et d'Histoire d'Antibes). APDCA, Juin-les-Pains, pp. 195-209.

Fernandes, P., Raynal, J.P., Moncel, M.H., 2008. Middle Palaeolithic raw material gathering, territories and human mobility in the southern Massif Central, France. First results from a petro-archaeological study on flint. Journal of Archaeological Science 35, 2367-2370. 
Ferreira, A.de B., 1978. Planaltos e Montanhas do Norte da Beira: Estudo de Geomorfologia. Centro de Estudos Geográficos, Lisboa.

Gabriel, S., Béarez, P., 2009. Caçadores-pescadores do vale do Côa: Os restos de fauna do sítio do Fariseu. In: Aubry, T. (Ed.), 200 Séculos de História do Vale do Côa: Incursões na Vida Quotidiana dos Caçadores-Artistas do Paleolítico. IGESPAR, I.P. (Trabalhos de Arqueologia 52), Lisboa, pp. 331-339.

Gamble, C., 1999. The Palaeolithic Societies of Europe. Cambridge University Press, Cambridge.

Gameiro, C., Aubry, T., Almeida, F., 2008. L'Exploitation des matières premières lithiques au Magdalénien final en Estremadure Portugaise: Données sur les sites de Lapa dos Coelhos et de l'abri 1 de Vale dos Covões. In: Aubry, T., Almeida, F., Araújo, A.C. Tiffagom, M. (Eds.), Space and Time: Which Diachronies, which Synchronies, which Scales?/Typology vs. Technology. Archaeopress (BAR International Series 1831), Oxford, pp. 57-67.

García Díez, M., Aubry, T., 2002. Grafismo mueble en el valle del Côa (Vila Nova de Foz Côa, Portugal). Zephyrus 55, 157-182.

Geneste, J.M., 1985. Analyse lithique d'industries moustériennes du Périgord: Une approche technologique du comportement des groupes humains au Paléolithique moyen. PhD Thesis. University of Bordeaux I.

Gould, R.A., Saggers, S., 1985. Lithic procurement in Central Australia: a closer look at Binford's idea of embeddedness in archaeology. Antiquity 50 (1), 117-136.

Grootes, P.M., Stuiver, M., 1997. Oxygen 18/16 variability in Greenland snow and ice with $10-3$ to 105 -year time resolution. Journal of Geophysics Research 102 . 26455-26470.

Guy, E., 2002. Contribution de la stylistique à l'estimation chronologique des piquetages paléolithiques de la Vallée du Côa (Portugal). In: Sacchi, D. (Ed.), L'art paléolithique à l'air libre: le paysage modifié par l'image (Tautavel, Campôme, 7-9 octobre 1999). GAEP; GÉOPRE, Saint-Estève, pp. 65-72.

Hamilton, M.J., Milne, B.T., Walker, R.S., Brown, J.H., 2007. Nonlinear scaling of space use in human hunter-gatherers. Proceedings of the National Academy of Science 104 (11), 4765-4769.

Haws, J., 2011. Paleolithic socionatural relationships during MIS 3 and 2 in central Portugal. Quaternary International. http://dx.doi.org/10.1016/ j.quaint.2011.10.003.

Heinrich, H., 1988. Origin and consequences of cyclic ice rafting in the northeast Atlantic ocean during the past 130,000 years. Quaternary Research 29, 142-152.

Hertell, E., Tallavaara, M., 2011. High mobility or gift exchange: Early Mesolithic exotic chipped lithics in Southern Finland. In: Rankama, T. (Ed.), Mesolithic Interfaces: Variability in Lithic Technologies in Eastern Fennoscandia. Archaeological Society of Finland (Monographs of the Archaeological Society of Finland 1), Saarijärvi, pp. 10-41.

Hill, K.R., Walker, R.S., Božičević, M., Eder, J., Headland, T., Hewlett, B., Magdalena Hurtado, A., Marlowe, F., Wiessner, P., Wood, B., 2011. Co-residence patterns in hunter-gatherer societies show unique human social structure. Science 331, 1286-1289.

Hockett, B., Bicho, N.F., 2000. The rabbits of Picareiro Cave: small mammal hunting during the Late Upper Paleolithic in the Portuguese Estremadura. Journal of Archaeological Science 27, 715-723.

Hockett, B., Haws., J., 2009. Continuity in Animal Resource Diversity in the Late Pleistocene Human Diet of Central Portugal. Before Farming 2009/2, Article 2.

Ichikawa, M., 1978. The residential groups of the Mbuti pygmies. Senri Ethnological Studies 1, 131-188.

IGME (Instituto Geológico y Minero de España), 1982. Mapa Geológico de España. E. 1,50,000: Cigales. Hoja 343, 16-14. Servicio de Publicaciones del Ministerio de Industria e Energía, Madrid.

Imhof, E., 1950. Gelände und Karte. Eugen Rentsch Verlag, Erlenbach; Zurich.

Kelly, R.L., 1992. Mobility/Sedentism: concepts, archaeological measures and effects. Annual Review of Anthropology 21, 43-66.

Kelly, R.L., 1995. The Foraging Spectrum: Diversity in Hunter-Gatherer Lifeways. Smithsonian Institution Press, Washington.

Kelly, R.C., 2005. The evolution of lethal intergroup violence. Proceedings of the National Academy of Science 102, 15294-15298.

Klaric, L., Guillermin, P., Aubry, T., 2009. Des armatures variées et des modes de productions variables: Réflexions à partir de quelques exemples issus du Gravettien d'Europe occidentale (France, Portugal, Allemagne). Gallia Préhistoire 51, 113-154.

Kullberg, J.C., Rocha, R.B., Soares, A.F., Rey, J., Terrinha, P., Callapez, P., Martins, L., 2006. A Bacia Lusitaniana: Estratigrafia, paleogeografia e tectónica. In: Dias, R., Araújo, A., Terrinha, P., Kullberg, J.C. (Eds.), Geologia de Portugal no Contexto da Ibéria. Universidade de Évora, Évora, pp. 317-368.

Lambeck, K., Chappell, J., 2001. Sea level change through the last glacial cycle. Science 292, 679-686.

Laming-Emperaire, A., 1972. Art rupestre et organisation sociale. In: Almagro Bash, M., García Guinea, M.A. (Eds.), Santander Symposium. Santander, Madrid, UISPP, pp. 65-79.

Le Brun Ricalens, F., 2006. Les pièces esquillées: État des connaissances après un siècle de reconnaissance. Paleo 18, 95-114.

Lebreiro, S.M., Moreno, J.C., Mac Cave, I.N., Weaver, P.P.E., 1995. Evidence of Heinrich event layers of Portugal (Tore Seamount, 39 N, 12 W). Marine Geology 131, 47-56.

Lee, R.B., 1972. The intensification of social life among ! Kung Bushmen. In: Spooner, B. (Ed.), Population Growth: Anthropological Implications. MIT Press, Cambridge, pp. 343-350.

Lee, R.B., Daly, R., 1999. Foragers and others. In: Lee, R.B., Daly, R. (Eds.), The Cambridge Encyclopedia of Hunter and Gatherers. Cambridge University Press, Cambridge, pp. 1-19.
Leroi-Gourhan, A., Brézillon, M., 1966. L'habitation magdalénienne n 1 de Pincevent près Montereau (Seine-et-Marne). Gallia Préhistoire 9 (2), 263-365.

Macdonald, D.H., 1998. Subsistence, sex, and cultural transmission in Folsom Culture. Journal of Anthopological Archaeology 17, 217-239.

Mangado Llach, X., 2002. La Caracterización y el Aprovisionamiento de los Recursos Abióticos en la Prehistoria de Cataluña: Las Materias Primas Silíceas del Paleolítico Superior Final y el Epipaleolítico. PhD Thesis. University of Barcelone.

Mangado Llach, X., 2004. L’Arqueopetrologia del Sílex. Una Clau pel Coneixement Paleoeconòmic i Social de les Poblacions Prehistòriques. Societat Catalana d'Arqueologia, Barcelona.

Mauss, M., Beuchat, S., 1905. Essai sur les variations saisonnières des sociétés eskimo: Étude de morphologie sociale. Année Sociologique 9, 39-132.

McDougall, N., Brenchley, P.J., Rebelo, J.A., Romano, M., 1987. Fans and fan deltas: precursors to the Armorican Quartzite (Ordovician) in western Iberia. Geological Magazine 124, 347-359.

Mediavilla, R.M., Dabrio, C.J., 1986. La sedimentación continental del Neógeno en el sector Centro-septentrional de la depresión del Duero (provincia de Palencia) Studia Geologia Salmanticensia 32, 111-132.

Mercier. N., Valladas, H., Froget, L., Jorons, J.-L., Aubry, T., 2001. Application de la méthode de la thermoluminescence à la datation des occupations paléolithiques de la vallée du côa. In: Zilhão, J., Aubry, T., Carvalho, A.F. (Eds.), Les premiers hommes modernes de la péninsule ibérique (Actes du Colloque de la Commission VIII de l'UISPP, Vila Nova de Foz Côa, 22-24 octobre 1998). Instituto Português de Arqueologia (Trabalhos de Arqueologia 17), Lisboa, pp. 275-280.

Mercier, N., Valladas, H., Aubry, T., Zilhão, J., Jorons, J.-L., Reyss, J.-L., Sellami, F. 2006. Fariseu: First confirmed open-air Palaeolithic parietal art site in the Côa Valley (Portugal). Antiquity 80 (310) Project Gallery. <http://antiquity.ac.uk/ projgall/mercier $>$ (accessed 10.01.12)

Molina Ballesteros, E., García Talegón, G., Vicente Hernández, M.A., 1997. Estudio de perfiles silicificados en el borde SO de la cuenca terciaria del Duero: Una nueva interpretación genética de la silicificación. Revista de la Sociedad Geológica de España 10 (3-4), 327-338.

Owens, D., Hayden, B., 1997. Prehistoric rites of passage: a comparative study of transegalitarian hunter-gatherers. Journal of Anthropological Archaeology 16, 121-161.

Pelegrin, J., 2000. Les techniques de débitage laminaire au Tardiglaciaire: Critères de diagnose et quelques réflexions. In: Valentin, B., Bodu, P., Christensen, M. (Ed.) L'Europe Centrale et Septentrionale au Tardiglaciaire: Confrontation des Modèles Régionaux de Peuplement. Éditions de l'APRAIF (Mémoire du Musée de Préhistoire d'île-de-France 7), Nemours, pp. 73-86.

Pereira, T., 2010. A Exploração do Quartzito na Faixa Atlântica Peninsular no Final do Plistocénico. PhD Thesis. University of Algarve.

Peterson, N., 1993. Demand sharing: reciprocity and the pressure for generosity among foragers. American Anthropologist 95 (4), 860-874.

Polanyi, K., 1957. The economy as instituted process. In: Polanyi, K., Arensberg, C., Pearson, H. (Eds.), Trade and Market in the Early Empires. Free Press, New York, pp. 243-270.

Proença Cunha, P., 2000. Paleoalterações e cimentações nos depósitos continentais terciários de Portugal central: Importância na interpretação de processos antigos. Ciências da Terra 14, 145-154.

Renfrew, C., 1975. Trade as action at a distance: questions of integration and communication. In: Sabloff, J.A., Lamberg-Karlovsky, C.C. (Eds.), Ancient Civilization and Trade. University of New Mexico Press, Albuquerque, pp. 3-60.

Ribeiro, A., Antunes, M.T., Ferreira, M.P., Rocha, M.P., Soares, A.F., Zbyszewski, G., Moitinho de Almeida, F., Carvalho, D., Monteiro, J.H., 1979. Introduction à la géologie générale du Portugal. Serviço Geológico de Portugal, Lisboa.

Roucoux, K.H., Shackelton, N.J., Abreu, L., 2001. Combined marine proxy and pollen analyses reveal rapid Iberian vegetation response to north Atlantic millennialscale climate oscillations. Quaternary Research 56, 128-132.

Roucoux, K.H., Abreu, L., Shackelton, N.J., Tzedakis, P.C., 2005. The response of NW Iberian vegetation to North Atlantic climate oscillations during the last $65 \mathrm{kyr}$ Quaternary Science Reviews 24, 1637-1653.

Sahlins, M., 1972. Stone Age Economics. Aldine, Chicago.

Sánchez Goñi, M.F., Turon, J.L., Eynaud, F., Gendreau, S., 2000. European 679 climatic response to milennial-scale changes in the atmosphere-ocean system during the last glacial period. Quaternary Research 54, 394-403.

Sánchez Goñi, M.F., Cacho, I., Turon, J.L., Guiot, J., Sierro, F.J., Peypouquet, J.P. Grimalt, J.O., Shackleton, N.J., 2002. Synchroneity between marine and terrestrial responses to millennial scale climate variability during the last glacial period in the Mediterranean region. Climate Dynamics 19, 95-105.

Sánchez Goñi, M.F., Landais, A., Fletcher, J.W., Naughton, F., Desprat, S., Duprat, J. 2008. Contrasting impacts of Dansgaard-Oeschger events over a western European latitudinal transect modulated by orbital parameters. Quaternary Science Reviews 27, 1136-1151.

Santos, A.T., in press. Reflexões sobre a arte paleolítica do Côa a propósito da superação de uma persistente dicotomia conceptual. In: I Mesa Redonda Artes Rupestres da Pré-História e da Proto-história (Proceedings).

Shackleton, N.J., Hall, M., Vincent, E., 2000. Phase relationship between millennialscale events 64000-24000 years ago. Paleoceanography 15, 565-569.

Skinner, L.C., Elderfield, H., 2007. Rapid fluctuations in the deep North Atlantic heat budget during the last glacial period. Paleoceanography 22, 1-9.

Steward, J., 1936. The economic and social basis of primitive bands. In: Lowie, R. (Ed.), Essays in Anthropology Presented to A.L. Kroeber. University of California Press, Berkeley, pp. 331-350. 
Thouveny, N., Moreno, E., Delanghe, D., Candon, L., Lancelot, Y., Shackleton, N.J. 2000. Rock magnetic detection of distal ice-rafted debris: clue for the identification of Heinrich layers on the Portuguese margin. Earth and Planetary Science Letters 180, 61-75.

Tobler, W. 1993 Three Presentations on Geographical Analysis and Modeling.Technical Report 93-1. National Center for Geographic Information and Analysis. <http://www.ncgia.ucsb.edu/Publications/Tech_Reports/93/931.PDF> (accessed 28.07.11).

Tripcevich, N., 2007. Quarries, Caravans, and Routes to Complexity: Prehispanic Obsidian in the South-Central Andes. PhD Thesis. UC Santa Barbara Anthropology. <http://works.bepress.com/tripcevich/8> (accessed 28.07.11).

Tripcevich, N., 2009. II. Cost-Distance Analysis. <http://mapaspects.org/node/3744> (accessed 12.01.12).

Turon, J.-L., Lézine, A.-M., Denèfle, M., 2003. Land-sea correlation for the last glaciations inferred from a pollen and dinocyst record from the Portuguese margin. Quaternary Research 59, 88-96.

Valladas, H., Mercier, N., Froget, L., Jorons, J.-L., Reyss, J.-L., Aubry, T., 2001. TL dating of Upper Palaeolithic sites in the Côa Valley (Portugal). Quaternary Science Reviews 20, 939-943.

van Leusen, P.M., 2002. Pattern to Process: Methodological Investigations into the Formation and Interpretation of Spatial Patterns in Archaeological Landscapes. PhD Thesis. Univ. Groningen. <http://irs.ub.rug.nl/ppn/239009177> (accessed 23.09.11).

Walker, R.S., Hill, K.R., Flinn, M.V., Ellsworth, R.M., 2011. Evolutionary history of hunter-gatherer marriage practice. PLoS ONE 6 (4), e19066. http://dx.doi.org/ 10.1371/journal.pone.0019066.

Whallon, R., 2006. Social networks and information: non "utilitarian" mobility among hunter-gatherers. Journal of Anthropological Archaeology 25, 259-270.

Wheatley, D., Gillings, M., 2002. Spatial Technology and Archaeology: The Archaeological Applications of GIS. Taylor \& Francis, New York.
Wilson, R.C., Hiscott, R.N., Willis, M.G., Gradstein, F.M., 1989. The Lusitanian Basin of west-central Portugal: Mesozoic and Tertiary tectonic, stratigraphic and subsidence history. In: Tankard, A.J., Balkwill, H. (Eds.), Extensional Tectonics and Stratigraphy of the North Atlantic Margins. American Association of Petroleum Geologists (Memoirs 46), Tulsa, pp. 341-361.

Winterhalder, B., 2001. The behavioural ecology of hunter gatherers. In: PanterBrick, C., Layton, R.H., Rowley-Conwy, P. (Eds.), Hunter-Gatherers: An Interdisciplinary Perspective. Cambridge University Press, Cambridge, pp. 1238.

Wobst, H.M., 1974. Boundary conditions for Paleolithic social systems: a simulation approach. American Antiquity 39 (2), 147-178.

Wobst, H.M., 1977. Stylistic behavior and information exchange. In: Cleland, E.H. (Ed.), For the Director: Research Essays in Honor of James B. Griffen. University of Michigan Museum of Anthropology (Anthropological Papers), Ann Arbour, pp. 317-342.

Zahn, R., Schoenfeld, J., Kudrass, H.R., Park, M.H., Erlenkeuser, H., Grootes, P., 1997 Thermohaline instability in the North Atlantic during meltwater events: stable isotopes and ice-rafted detritus records from Core SO75-26KL. Paleoceanography $12,696-710$.

Zilhão, J., 1997. O Paleolítico Superior da Estremadura Portuguesa. Edições Colibri, Lisboa.

Zilhão, J., 2003. Vers une chronologie plus fine du cycle ancien de l'art paléolithique de la Côa: quelques hypothèses de travail. In: de Balbín Berhrmann, R., Bueno Ramírez, P. (Eds.), El Arte Prehistórico desde inicios del siglo XXI (Primer Symposium Internacional de Arte Prehistórico de Ribadesella). Ribadesella, Asociación de Amigos de Ribadesella, pp. 75-87.

Zilhão, J., Almeida, F., Aubry, T., Carvalho, A.F., Zambujo, G., 1995. O sítio arqueológico paleolítico do Salto do Boi (Cardina 1, Santa Comba, Vila Nova de Foz Côa). Trabalhos de Antropologia e Etnologia 35, 471-497. 